\title{
Vascular endothelial growth factor receptor-3 is a novel target to improve net ultrafiltration in methylglyoxal-induced peritoneal injury
}

\author{
Takeshi Terabayashi ${ }^{1}$, Yasuhiko Ito ${ }^{1}$, Masashi Mizuno ${ }^{1}$, Yasuhiro Suzuki ${ }^{1}$, Hiroshi Kinashi ${ }^{1}$, Fumiko Sakata $^{1}$, \\ Takako Tomita ${ }^{1}$, Daiki Iguchi ${ }^{1}$, Mitsuhiro Tawada ${ }^{1}$, Ryosuke Nishio ${ }^{2}$, Shoichi Maruyama' ${ }^{1}$, Enyu Imai ${ }^{1,3}$, Seiichi Matsuo ${ }^{1}$ \\ and Yoshifumi Takei ${ }^{4}$
}

Appropriate fluid balance is important for good clinical outcomes and survival in patients on peritoneal dialysis. We recently reported that lymphangiogenesis associated with fibrosis developed in the peritoneal cavity via the transforming growth factor- $\beta 1$-vascular endothelial growth factor-C (VEGF-C) pathway. We investigated whether VEGF receptor-3 (VEGFR-3), the receptor for VEGF-C and -D, might be a new target to improve net ultrafiltration by using adenovirusexpressing soluble VEGFR-3 (Adeno-sVEGFR-3) in rodent models of peritoneal injury induced by methylglyoxal (MGO). We demonstrated that lymphangiogenesis developed in these MGO models, especially in the diaphragm, indicating that lymphangiogenesis is a common feature in the peritoneal cavity with inflammation and fibrosis. In MGO models, VEGF-D was significantly increased in the diaphragm; however, VEGF-C was not significantly upregulated. Adeno-sVEGFR-3, which was detected on day 50 after administration via tail vein injections, successfully suppressed lymphangiogenesis in the diaphragm and parietal peritoneum in mouse MGO models without significant effects on fibrosis, inflammation, or neoangiogenesis. Drained volume in the peritoneal equilibration test using a $7.5 \%$ icodextrin peritoneal dialysis solution (the 7.5\% icodextrin peritoneal equilibration test) was improved by Adeno-sVEGFR-3 on day $22(P<0.05)$ and day 50 after reduction of inflammation $(P<0.01)$, indicating that the $7.5 \%$ icodextrin peritoneal equilibration test identifies changes in lymphangiogenesis. The solute transport rate was not affected by suppression of lymphangiogenesis. In human peritoneal dialysis patients, the dialysate to plasma ratio of creatinine positively correlated with the dialysate VEGF-D concentration $(P<0.001)$. VEGF-D mRNA was significantly higher in the peritoneal membranes of patients with ultrafiltration failure, indicating that VEGF-D is involved in the development of lymphangiogenesis in peritoneal dialysis patients. These results indicate that VEGFR-3 is a new target to improve net ultrafiltration by suppressing lymphatic absorption and that the $7.5 \%$ icodextrin peritoneal equilibration test is useful for estimation of lymphatic absorption.

Laboratory Investigation (2015) 95, 1029-1043; doi:10.1038/labinvest.2015.87; published online 29 June 2015

Maintenance of optimal fluid balance is important for good clinical outcomes and survival in patients who undergo peritoneal dialysis. Overhydration states, which are often associated with higher peritoneal transport rates, are reported to be a major cause for dialysis discontinuation. ${ }^{1,2}$ The pathological features of peritoneal membrane injury in peritoneal dialysis patients with high solute transport rates are submesothelial fibrosis, accumulation of extracellular matrix, and neoangiogenesis. ${ }^{3,4}$
We recently showed that lymphangiogenesis and vascular endothelial growth factor-C (VEGF-C) expression, one of the key mediators of lymphangiogenesis, were associated with fibrosis using human tissues, peritoneal dialysis effluent samples, cultured cells derived from peritoneal dialysis effluent, and rat chlorhexidine gluconate -induced peritoneal injury models. ${ }^{5}$ In those studies, we found that the VEGF-C content in the peritoneal dialysis effluent correlated with the peritoneal membrane transport rate and transforming growth

${ }^{1}$ Department of Nephrology and Renal Replacement Therapy, Nagoya University Graduate School of Medicine, Nagoya, Japan; ${ }^{2}$ Department of Emergency Medicine, Kyoto University Hospital, Kyoto, Japan; ${ }^{3}$ Nakayamadera Imai Clinic, Takarazuka, Japan and ${ }^{4}$ Department of Biochemistry, Nagoya University Graduate School of Medicine, Nagoya, Japan

Correspondence: Professor Y Ito, MD, PhD, Department of Nephrology and Renal Replacement Therapy, Nagoya University Graduate School of Medicine, 65 Tsurumai-cho, Showa-ku, Nagoya 466-8550, Japan.

E-mail: yasuito@med.nagoya-u.ac.jp

Received 28 September 2014; revised 30 April 2015; accepted 18 May 2015 
factor-beta (TGF- $\beta$ ) concentration, and that expression of VEGF-C and markers of lymphatics was higher in ultrafiltration failure-peritoneum. ${ }^{5}$ In cultured mesothelial cells and macrophages, TGF- $\beta$-induced VEGF-C expression was blocked by a TGF- $\beta$ type I receptor (TGF $\beta$ RI) inhibitor. ${ }^{5}$ Furthermore, VEGF-C expression and lymphangiogenesis were suppressed by the TGF $\beta$ RI inhibitor or by the cyclooxygenase-2 (COX-2) inhibitor, celecoxib, in chlorhexidine gluconate-induced peritoneal injury models. ${ }^{5}$ Thus, we proposed that lymphangiogenesis in the peritoneal membrane, similar to renal fibrosis is linked with the fibrotic process via the TGF- $\beta$-VEGF-C pathway. ${ }^{6-8}$ The lymphatic absorption rate, which is measured by the rate at which intraperitoneally administered radioactive serum albumin or the macromolecule dextran 70 disappears, is significantly higher in patients with ultrafiltration failure, and lymphatic absorption is considered to be one of the causes of the decrease in net ultrafiltration. ${ }^{9-12}$ However, the results from these clinical approaches have been controversial, ${ }^{13,14}$ and there are no other methods available to assess lymphatic function.

In the present study, we investigated whether VEGF receptor-3 (VEGFR-3), the receptor for VEGF-C and -D, might be a new target to increase net ultrafiltration by suppression of lymphangiogenesis using an adenovirusexpressing soluble VEGFR-3 (Adeno-sVEGFR-3) fused with human IgG (Supplementary Figure 1) in models of murine peritoneal injury induced by methylglyoxal (MGO). ${ }^{15-17}$ MGO is a precursor of advanced glycation end products, which accumulate in dialysis patients. ${ }^{18}$ In addition, we proposed a new method for the peritoneal equilibration test by using a $7.5 \%$ icodextrin peritoneal dialysis solution $(7.5 \%$ icodextrin peritoneal equilibration test) to assess lymphatic absorption (Supplementary Figure 1). Finally, we studied the expression of VEGF-D in lymphangiogenesis in human samples.

\section{MATERIALS AND METHODS MGO-Induced Peritoneal Injury Model}

All animal studies were carried out in accordance with the Animal Experimentation Guidelines of Nagoya University Graduate School of Medicine (Nagoya, Japan). Ten-week-old male C57BL/6J mice (Japan SLC, Hamamatsu, Japan) initially weighing $24-29 \mathrm{~g}$ were used throughout the study. The animals were maintained under conventional laboratory conditions and had free access to food and water. The mice received an intraperitoneal injection of $100 \mathrm{ml} / \mathrm{kg}$ body weight of peritoneal dialysis fluid (Dianeal-N PD-4-2.5, $2.27 \%$ glucose, Baxter, Tokyo, Japan) containing $20 \mathrm{mmol} / \mathrm{l}$ MGO (MP Biomedicals LLC, Illkirch, France) for 3 weeks, 5 consecutive days per week as described previously ${ }^{17}$ (Experiment 1, Supplementary Figure 2a). Control mice received the same dosage of peritoneal dialysis fluid without MGO. This peritoneal dialysis fluid was prepared by purification through a $0.2-\mu \mathrm{m}$ pore-size filter and by adjustment to $\mathrm{pH} 5.0$ immediately before injection every day. The mice were killed on day 22 , and parietal peritoneal and diaphragmatic samples were procured. The harvested samples were used for immunohistochemical analysis of lymphatic vessel endothelial hyaluronan receptor-1 (LYVE-1), VEGFR-3, CD31, type III collagen, CD68, and VEGF-D, and for analysis of the mRNA expression of VEGF-D, VEGFR-3, LYVE-1, and CD31.

\section{MGO Model Mice and Studies of Lymphangiogenesis Inhibition using an Adenovirus Vector}

The experimental design and protocols are as follows (Supplementary Figure 2):

(a) Experiment 1: development of a MGO-induced peritoneal injury model in mice. The mice received peritoneal dialysis fluid $(100 \mathrm{ml} / \mathrm{kg})$ with $\mathrm{MGO}(20 \mathrm{mM})$ intraperitoneally from day 1 until day 22 (Supplementary Figure 2a).

(b) Experiment 2: inhibition studies using an adenovirusexpressing sVEGFR-3 and a control adenovirusexpressing Lac $\mathrm{Z}$ in a MGO-induced peritoneal injury model. Recombinant Adeno-sVEGFR-3 or $\beta$-galactosidase (Adeno-LacZ) were kindly provided by K Alitalo (the University of Helsinki, Helsinki, Finland) and were amplified and purified for use as described previously. ${ }^{19-21}$ On day 0 , prior to establishment of MGO-induced peritoneal injury as in a, the mice were first administered $1.0 \times 10^{9}$ p.f.u. of one of the adenoviral vectors intravenously through the tail vein. On day 22 , the mice were assessed using a conventional peritoneal equilibration test with a $4.25 \%$ glucose-based peritoneal dialysis fluid and a peritoneal equilibration test with 7.5\% icodextrin (Adeno-LacZ Day 22 group and AdenosVEGFR-3 Day 22 groups; Supplementary Figure 2b).

(c) Experiment 3: development of a MGO-induced peritoneal injury model with peritoneal rest from day 22 to day 50. The mice were treated as in a until day 22 . From day 22 until their sacrifice on day 50 no dialysate was infused into the peritoneal cavity (peritoneal rest) (Supplementary Figure 2c).

(d) Experiment 4: inhibition studies using adenovirusexpressing sVEGFR-3 and control adenovirus-expressing Lac Z in a MGO model as in $\mathbf{b}$ except that the mice further underwent peritoneal lavage using a $1.5 \%$ peritoneal dialysis fluid from day 22 to day 50 . For this treatment, the mice received an intraperitoneal injection of $100 \mathrm{ml} / \mathrm{kg}$ body weight of a low concentration peritoneal dialysis fluid (Dianeal-N PD-4 1.5, 1.36\% glucose) for 20 days, 5 consecutive days per week. This procedure is similar to a previously reported method. ${ }^{22}$ In these mice, peritoneal functions were assessed on day 50 using a conventional peritoneal equilibration test with a $4.25 \%$ glucose-based peritoneal dialysis fluid and a peritoneal equilibration test with a $7.5 \%$ icodextrin peritoneal dialysis fluid (AdenoLacZ Day 50 group and Adeno-sVEGFR-3 Day 50 group; Supplementary Figure 2d).

Tissue and blood samples were also obtained from the mice for further analysis. 


\section{Histology and Immunohistochemistry}

Routine histological and immunohistological analyses of animal and human tissues were performed and assessed as we described previously. ${ }^{5-7,23,24}$ The antibodies used are listed in Supplementary Table 1. Mast cells were evaluated using sections stained with $0.2 \%$ toluidine blue.

\section{RNA Preparation from Peritoneal and Diaphragm Tissues, and PCR Analysis}

Animal peritoneal and diaphragm tissues were immersed in RNAlater (Ambion, Austin, TX, USA) for $>1$ day. RNA preparation and the synthesis of first-strand cDNA were performed as described previously. ${ }^{5-7,23,24}$ Total RNA $(1 \mu \mathrm{g})$ was then reverse transcribed. Quantitative real-time PCR (qPCR) analysis was performed with an Applied Biosystems (South San Francisco, CA, USA) Prism 7500HT sequence detection system using TaqMan gene expression assays as described previously. ${ }^{24}$ The TaqMan Gene Expression Assays (Applied Biosystems) used are described in Supplementary Table 2. 18S ribosomal RNA was used as an endogenous control. $5,7,23,24$

\section{Assessment of Lymphatic Absorption}

To assess absorption via the lymphatic vessels, animals that received $2000 \mu \mathrm{l}$ of $7.5 \%$ icodextrin peritoneal dialysis solution into the peritoneal cavity were killed at $4 \mathrm{~h}$ after infusion. An accurate drained volume was measured.

\section{Enzyme-Linked Immunosorbent Assays (ELISAs)}

The concentration of sVEGFR-3-Ig fusion protein in the serum was determined using an ELISA kit for human IgG1 (Cayman Chemical Company, Ann Arbor, MI, USA) as described previously. ${ }^{25}$ Levels of VEGF-D protein and prostaglandin E2 (PGE2) in the peritoneal dialysis effluent were measured using the ELISA kits for human VEGF-D (R\&D Systems, Minneapolis, MN, USA) and PGE2 (Cayman Chemical Company), respectively, according to the manufacturers' instructions. The samples were frozen at the time of collection and were stored at $-80^{\circ} \mathrm{C}$. The samples were not subjected to freeze-thaw cycles.

\section{Human Patient Studies}

All of the studies were approved by the Ethics Committee for Human Research of the Faculty of Medicine, Nagoya University (Approval \#298 and \#299). All patients provided informed consent prior to participation in this study.

\section{VEGF-D Concentration in the Peritoneal Effluent of Peritoneal Dialysis Patients}

The VEGF-D concentrations in dialysates were measured in overnight-dwelled $(8.95 \pm 1.63 \mathrm{~h})$ samples collected from 83 peritoneal dialysis patients ( 27 women and 56 men) who were treated between July 2005 and April 2008 in the Department of Nephrology and Renal Replacement Therapy of Nagoya University Hospital (Nagoya, Japan) and at affiliated hospitals. ${ }^{5}$ This is the same cohort in which VEGF-C was measured in recent studies. ${ }^{5}$ The mean age of all patients was $55.9 \pm 13.5$ (range, 28 to 89 ) years, and the mean duration of peritoneal dialysis treatment was $31.9 \pm 32.0$ (range, 1 to 132 ) months. ${ }^{5}$ Diabetic nephropathy was the cause of end-stage renal disease in 27 peritoneal dialysis patients (32.5\%). All patients were free from peritonitis for at least 1 month prior to the study, and patients with other diseases, such as liver or lung diseases and malignancy, were excluded. Patients undergoing combination therapy (hemodialysis+peritoneal dialysis) were not included in this study. Peritoneal membrane transport was assessed based on the dialysate to plasma ratio of creatinine, and the average value was $0.67 \pm 0.14$ (range, 0.28 to 0.96 ).$^{5} \mathrm{~A}$ fast peritoneal equilibration test was performed using a $2.27 \%$ glucose-based dialysis solution (Dianeal-N PD-4) as described by Twardowski et al (Supplementary Information 1). ${ }^{26}$ The correlation between VEGF-D concentration in the peritoneal dialysis effluent and the dialysate to plasma ratio of creatinine was analyzed. In addition, we measured the VEGF-D concentration in peritoneal effluent samples at $4 \mathrm{~h}$ of the peritoneal equilibration test. These samples were collected from 40 peritoneal dialysis patients ( 13 women and 27 men) treated between November 2008 and June 2009 at the Handa Municipal Hospital and the Nagoya University Hospital. The mean age of all patients was $52.9 \pm 10.9$ (range, 30 to 70 ) years, and the mean duration of peritoneal dialysis treatment was $26.1 \pm 24.6$ (range, 1 to 103 ) months. ${ }^{5,23}$

\section{VEGF-D mRNA Expression in the Human Peritoneum}

Fifty-four peritoneal tissue samples were obtained from 29 peritoneal dialysis patients and 25 pre-dialysis chronic renal failure patients at the time of peritoneal dialysis catheter insertion. Among the 29 peritoneal dialysis patients, 7 were regarded as having impaired ultrafiltration capacity, which was defined as described previously. ${ }^{5,23,27}$ Twenty-two patients (incident) had their catheters removed because of transplantation, severe exit site infection, mental disorders, or difficulty in performing the bag exchanges (Table 1).

\section{VEGF-D mRNA Expression in the Cultured Mesothelial Cells}

Reverse Transcription-PCR (RT-PCR) was performed using the HotStarTaq PCR kit (Qiagen, Tokyo, Japan) $)^{7,28}$ to examine whether VEGF-D mRNA was expressed in three kinds of mesothelial cells: the human mesothelial cell line Met5A, ${ }^{5,23}$ mesothelial cells from the peritoneal dialysis effluent of the patients on peritoneal dialysis, 5,23 and mesothelial cells derived from the omentum. ${ }^{29,30}$ The primers according to the reported sequences of human VEGF-D and GAPDH were 5'-GTATGGACTCTCGCTCAGCAT-3' (sense) and 5'-AGGCTCTCTTCATTGCAACAG-3' (antisense, PCR products $225 \mathrm{bp}) 3^{31}$ and $5^{\prime}$-ATCATCCCTGCCTCTACTGG-3' (sense) and 5'-CCCTCCGACGCCTGCTTCAC-3' (antisense, PCR products $188 \mathrm{bp}$ ), ${ }^{28}$ respectively. 


\section{Statistical Analyses}

The Shapiro-Wilk test was applied to test normal distributions. Values are expressed as mean \pm s.d. Differences between two groups were analyzed by the unpaired $t$-test or by the

Table 1 Profiles of peritoneal biopsy cases evaluated for VEGF-D mRNA expression

\begin{tabular}{lccc}
\hline & $\begin{array}{c}\text { Pre-dialysis } \\
\text { uremia }\end{array}$ & Incident & UFF \\
\hline$N$ & 25 & 22 & 7 \\
Male & 16 & 13 & 4 \\
Female & 9 & 9 & 3 \\
Age, years & $63.2 \pm 10.4$ & $58.5 \pm 14.1$ & $60.4 \pm 10.0$ \\
Duration of treatment, years & 0 & $3.7 \pm 3.0$ & $10.1 \pm 5.1$ \\
Average thickness of & $131.3 \pm 38.9$ & $148.1 \pm 90.5$ & $317.1 \pm 118.0$ \\
peritoneum, $\mu \mathrm{m}$ & & &
\end{tabular}

a LYVE-1
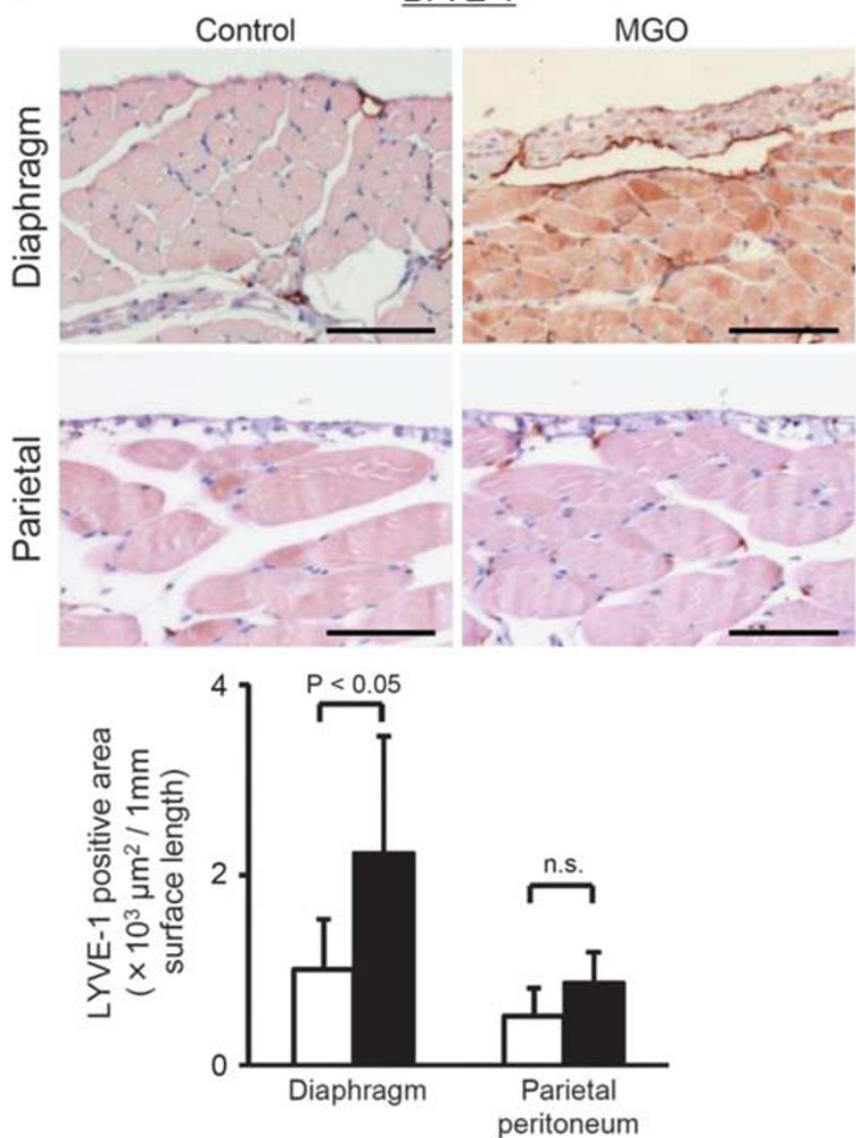

Mann-Whitney $U$-test. Comparisons among groups were performed by one-way analysis of variance with the Tukey's test, the Games-Howell test or the Dunnett's test, or by the Kruskal-Wallis multiple comparison test. Spearman's correlation coefficient was used to analyze correlations. Differences were considered to be statistically significant if $P<0.05$. All analyses were performed using SPSS software (SPSS, Chicago, IL, USA).

\section{RESULTS}

\section{Lymphangiogenesis Developed in a MGO-Induced Peritoneal Injury Model}

Twenty-two days after administration of MGO (Experiment 1, Supplementary Figure 2a) LYVE-1- and podoplaninpositive lymphatic vessels were increased and dilated in the diaphragm of MGO model mice compared with controls (Figure 1a,Supplementary Figure 3); however, lymphangiogenesis was not pronounced or significant in the parietal peritoneum wall (Figure 1a). In contrast, as shown in

b

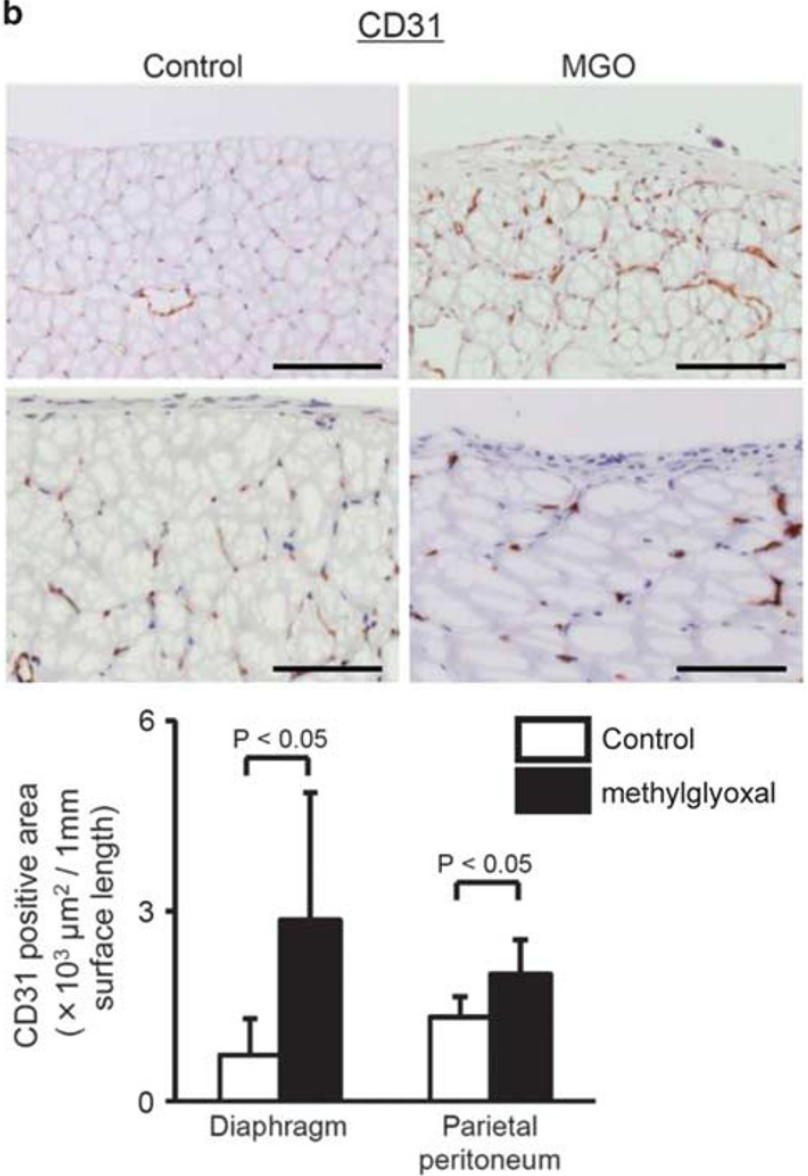

Figure 1 LYVE-1-positive lymphatic vessels were increased and dilated in the diaphragm of methylglyoxal mouse models compared with controls. Expression of lymphatic vessels was predominant in the diaphragm compared with that of control mice. Quantification of immunohistochemical parameters indicated the following: (a) LYVE-1-positive lymphatic vessels were significantly increased in the diaphragm, but not in the parietal peritoneal membrane; CD31-positive vessels (b), expression of type III collagen (c), and CD68-positive macrophages (d) were significantly increased in the diaphragm of methylglyoxal model mice compared with normal control mice. (each group, $n=6$ ). Scale bars, $100 \mu \mathrm{m}$. LYVE-1, lymphatic vessel endothelial hyaluronan receptor-1; NS, not significant. 
c

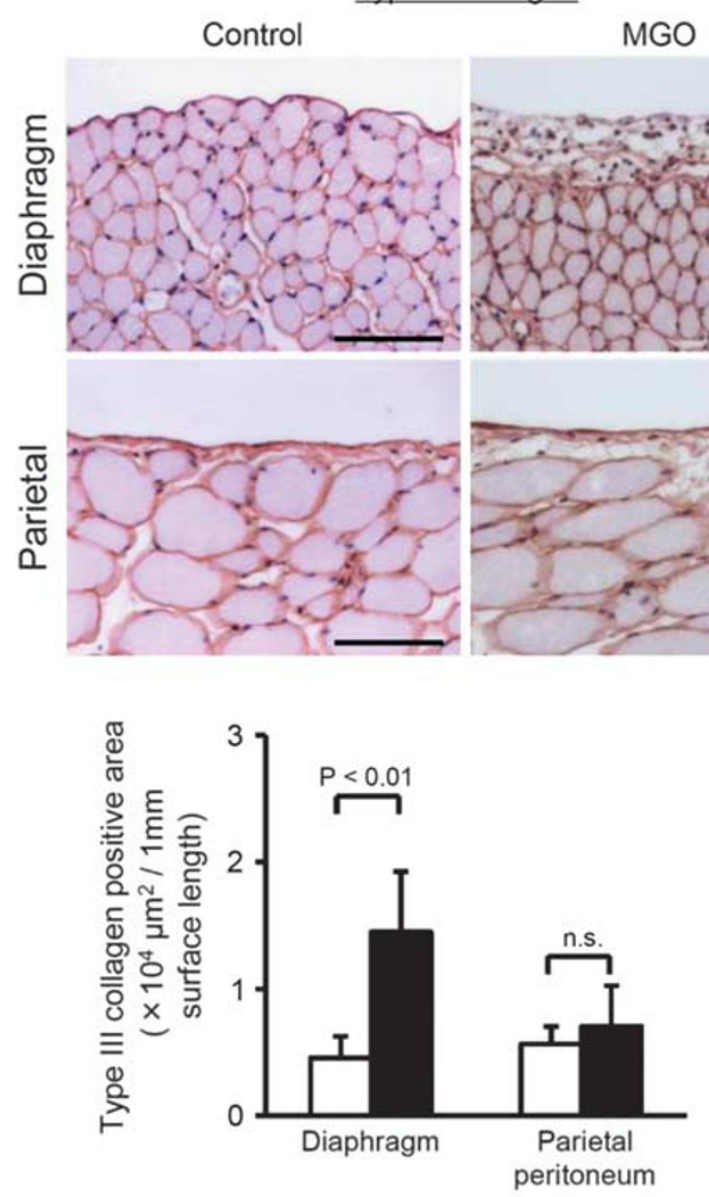

d
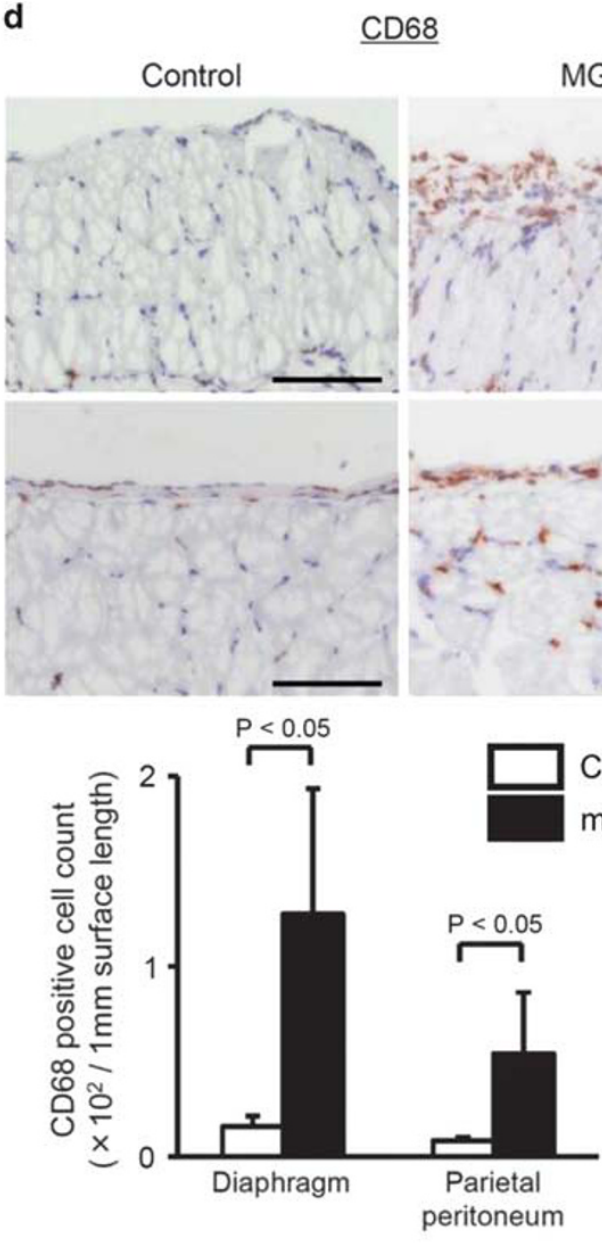

MGO
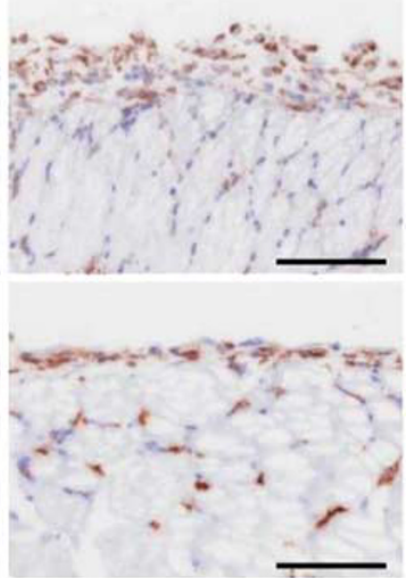

Control

methylglyoxal

Figure 1 Continued.

Supplementary Figure 4, in the advanced fibrotic area of the diaphragm entrances (red arrows) of newly developed lymphatic vessels were revealed in the fibrotic diaphragm. CD31-positive vessels, indicating neoangiogenesis $(P<0.05$; Figure $1 \mathrm{~b}$ ), expression of type III collagen, indicating fibrosis $(P<0.01$; Figure 1c), and CD68-positive macrophages, indicating inflammation $(P<0.05$; Figure $1 \mathrm{~d})$, were significantly increased in the diaphragm of the MGO models, but were not significantly increased in parietal peritoneal membranes. To confirm these immunohistochemical findings, we performed qPCR. Compared with normal mice, LYVE-1 $(P<0.01)$, VEGFR-3 $(P<0.001)$, and podoplanin mRNA $(P<0.001)$, which are typical markers of lymphatic endothelial cells, were significantly increased in the diaphragm of the MGO model mice (Figure 2, Supplementary Figure 3). Although VEGF-D mRNA and protein expression were upregulated in the diaphragm of MGO model mice, VEGF-C mRNA and protein were not significantly increased compared with normal mice (Figure 2, Supplementary Figure
5). Neither VEGF-C nor -D were significantly elevated in parietal peritoneum, (Figure 2, Supplementary Figure 5). VEGF-C and -D proteins were undetectable in the serum and peritoneal dialysis fluid. Interestingly, expression of lymphatic vessels markers is higher in the diaphragm than in parietal peritoneum (Figure 2, Supplementary Figure 5). We also developed rat MGO models and found that lymphangiogenesis developed in the diaphragm, and that VEGF-D expression in the diaphragm predominated over VEGF-C expression (Supplementary Figure 6). Double immunohistochemical staining indicated that the expression of LYVE-1-positive lymphatic vessels was similar to that of VEGFR-3-positive vessels (Figure 3a), and that VEGF-D was mainly expressed by CD68-positive macrophages in the MGO mouse model (Figure $3 \mathrm{~b}$ and $\mathrm{c}$ ), but not by mesothelial cells (data not shown). RT-PCR and ELISA using three different sources of cultured human mesothelial cells further suggested that neither VEGF-D mRNA (Figure 3d) nor protein (Figure 3e) is expressed by mesothelial cells. 
a

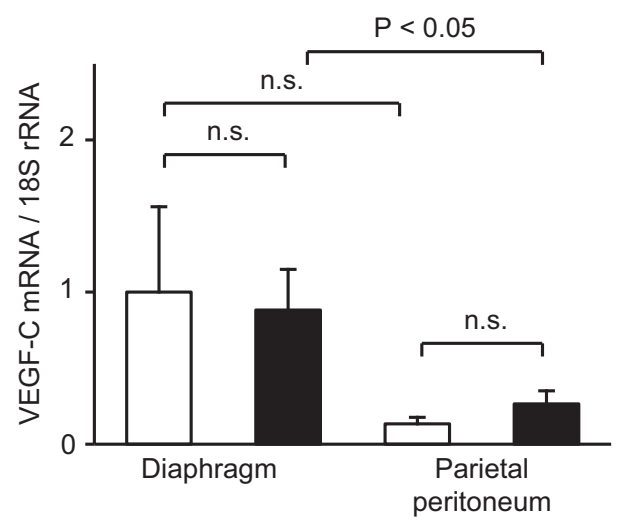

C

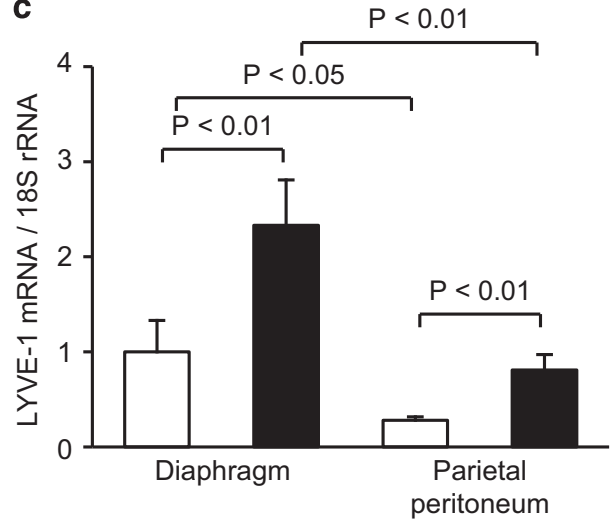

e

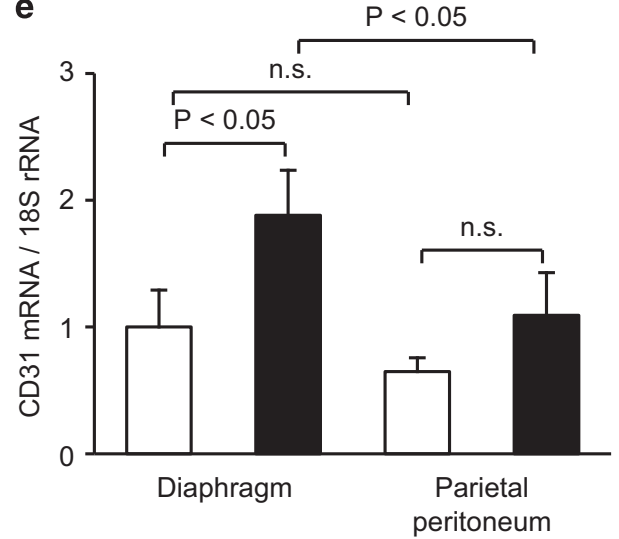

b

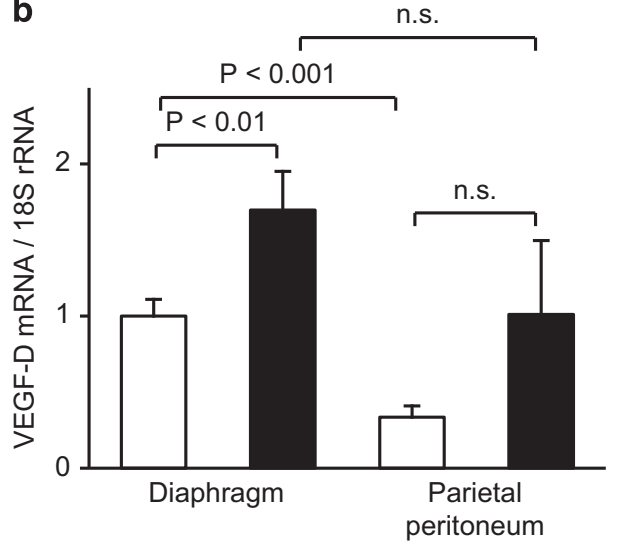

d

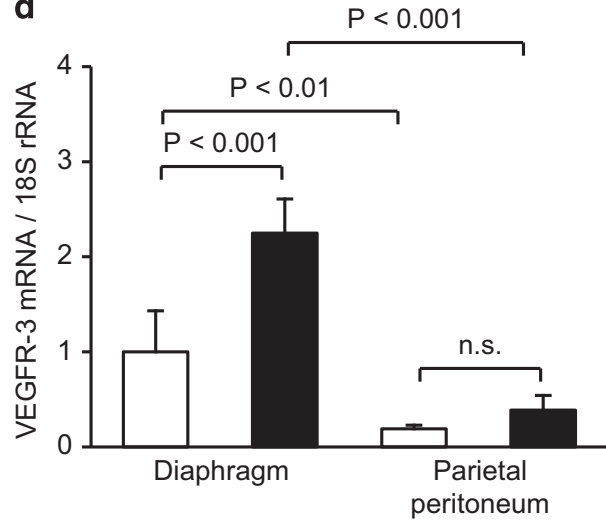

$\square$ Normal

methylglyoxal

Figure 2 Quantitative PCR analyses of specific mRNA expression in the diaphragm and parietal peritoneum of methylglyoxal model mice. VEGF-C mRNA expression was not significantly increased in the diaphragm (a). VEGF-D (b), LYVE-1 (c), VEGFR-3 (d), and CD31 (e) mRNA expression were increased in the diaphragm in the methylglyoxal model mice compared with normal controls. (each group, $n=6$ ). NS, not significant; VEGF, vascular endothelial growth factor.

Figure 3 Analysis of VEGF-D expression. (a-c) Representative double immunofluorescent staining for VEGFR-3 and LYVE-1 (a), and for CD68 and VEGF-D (b) in the diaphragm of a methylglyoxal mouse is shown. Arrows and arrowheads of the same color indicate the same cells. Scale bars, $50 \mu \mathrm{m}$. Quantification of CD68- and VEGF-D-stained cells (number of labeled cells per high power field (HPF)) in control and methylglyoxal mouse models is shown in c. VEGF-D was mainly expressed by CD68-positive macrophages in the methylglyoxal mouse model (c). $n=6$ in both control and methylglyoxal models. (d and e) VEGF-D mRNA (d) and protein (e) expression analyzed using RT-PCR and ELISA, respectively, were not detected in the human mesothelial cell line Met5A (d, lane 1) (e), in mesothelial cells derived from the peritoneal dialysis effluent of the patients on peritoneal dialysis (d, lane 2) (e), or in mesothelial cells from the omentum (d, lane 3) (e). In contrast, VEGF-D mRNA was detected in peritoneal tissues (d, lane 4) and VEGF-D protein was detected in peritoneal dialysis effluent (e) from patients with ultrafiltration failure (Table 1). (d) GAPDH was used as a loading control. PD, peritoneal dialysis, ND, not detected. 
sVEGFR-3 was Detected in Serum on Days 22 and 50 after Administration of the Adenovirus-Expressing sVEGFR-3

To investigate whether these occurrences of lymphangiogenesis were enhanced via VEGFR-3 activation, we performed inhibition studies using Adeno-sVEGFR-3, which traps both its VEGF-C and VEGF-D ligands. Serum sVEGFR-3 was detected on days 22 and 50 after its administration via tail vein injections, and its concentration was significantly higher on both days in mice injected with Adeno-sVEGFR-3 than in mice treated with Adeno-LacZ (Supplementary Figure 7). These results are consistent with previous reports. ${ }^{19,20,32}$ a

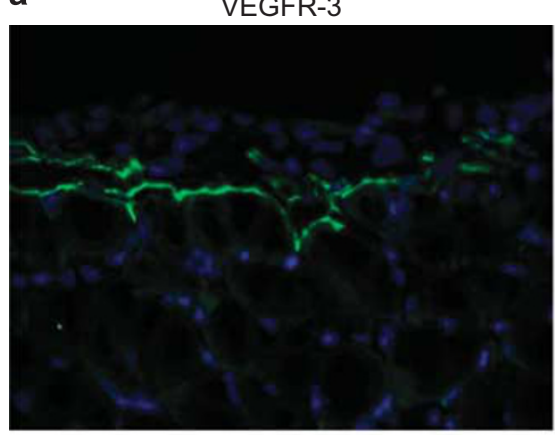

b

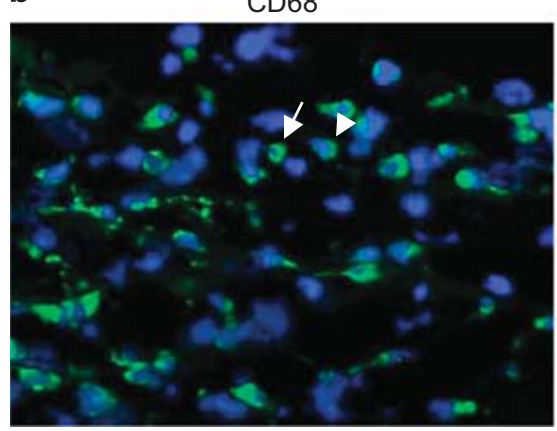

c

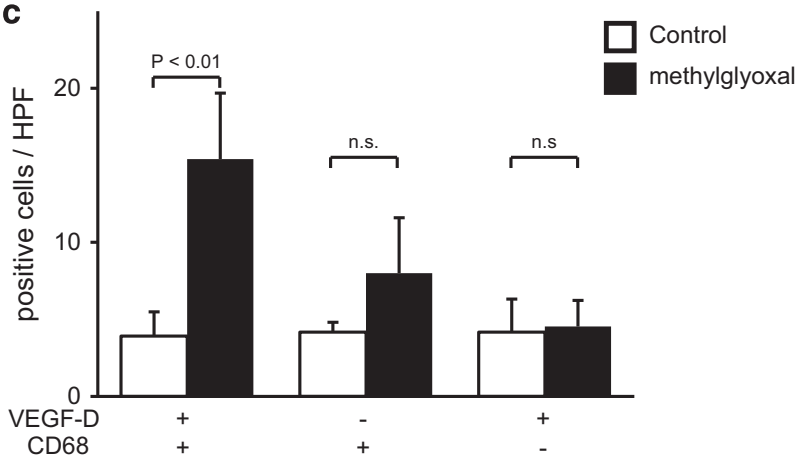

LYVE-1

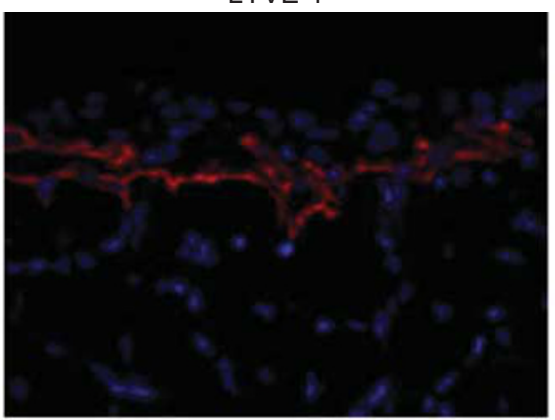

VEGF-D
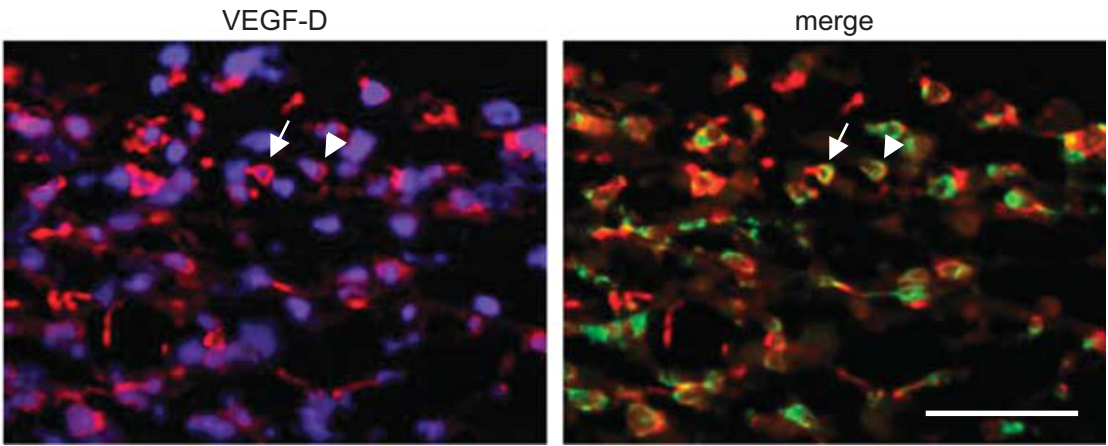

d

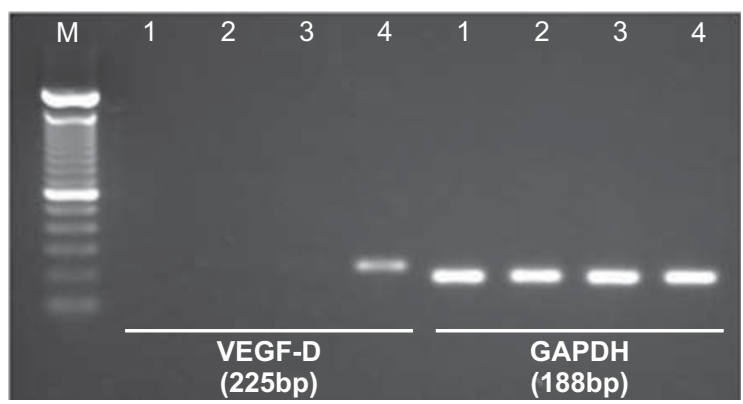

M: 100bp ladder

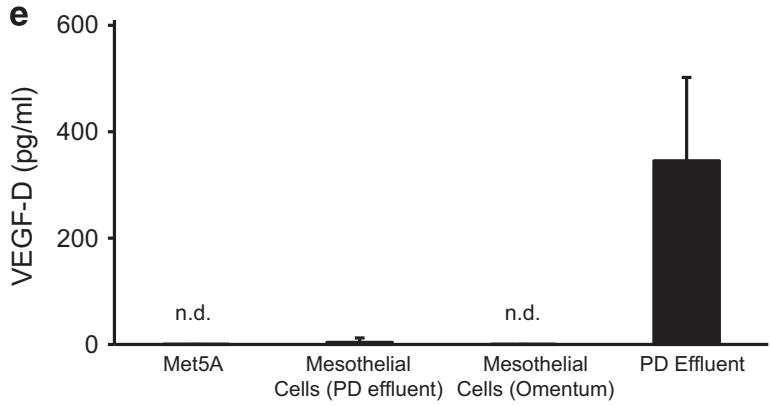




\section{Adenovirus-sVEGFR3 Suppressed Lymphangiogenesis in the Diaphragm and Parietal Peritoneal Membrane of MGO Model Mice on Day 22}

Expression of LYVE-1-positive lymphatic vessels in the diaphragm was significantly suppressed by Adeno-sVEGFR-3, but not by Adeno-LacZ (Figure 4a). However, there were no differences between mice treated with Adeno-sVEGFR-3 or Adeno-LacZ with respect to expression of CD31-positive vessels, type III collagen, or immune cells including CD68positive macrophages, Ly6B-positive granulocytes, $\mathrm{CD} 4$ or CD8 T cells, or CD11c dendritic cells (Figure 4b-d, Supplementary Figure 8). In addition, the percentage of VEGF-Dpositive macrophages was similar between the two groups (Supplementary Figure 9a). qPCR analysis indicated that, on day 22, LYVE-1 and VEGFR-3 mRNA were significantly suppressed by Adeno-sVEGFR-3 compared with Adeno-LacZ; however, neither VEGF-C nor VEGF-D mRNA or protein expression was significantly changed between the two groups
(Figure 5, Supplementary Figure 9b and c). VEGF-C and -D proteins were undetectable in the serum of both groups. The extent of neoangiogenesis and fibrosis as assessed by analysis of CD31 and TGF- $\beta 1$ mRNA levels was similar between the two groups (Figure 5e and f). These findings suggest that Adeno-sVEGFR-3 treatment specifically blocked the VEGFR-3 signaling pathway.

\section{Analysis of Lymphatic Absorption on Day 22}

To assess suppression of lymphangiogenesis by AdenosVEGFR-3, we performed a peritoneal equilibration test on day 22 using a $7.5 \%$ icodextrin peritoneal dialysis solution (Extraneal, Baxter), which is mainly absorbed via the lymphatic system. ${ }^{33}$ In this peritoneal equilibration test $(7.5 \%$ icodextrin peritoneal equilibration test), effluent volume was recovered in mice in which lymphangiogenesis was suppressed by administration of Adeno-sVEGFR-3 (Figure 6a), although angiogenesis as assessed by CD31 a

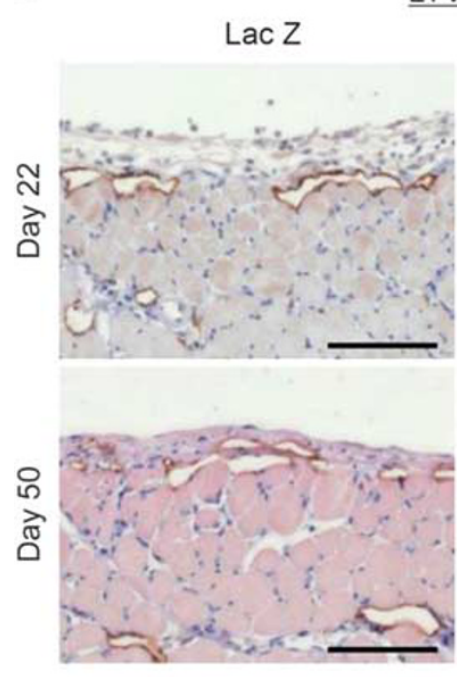

LYVE-1
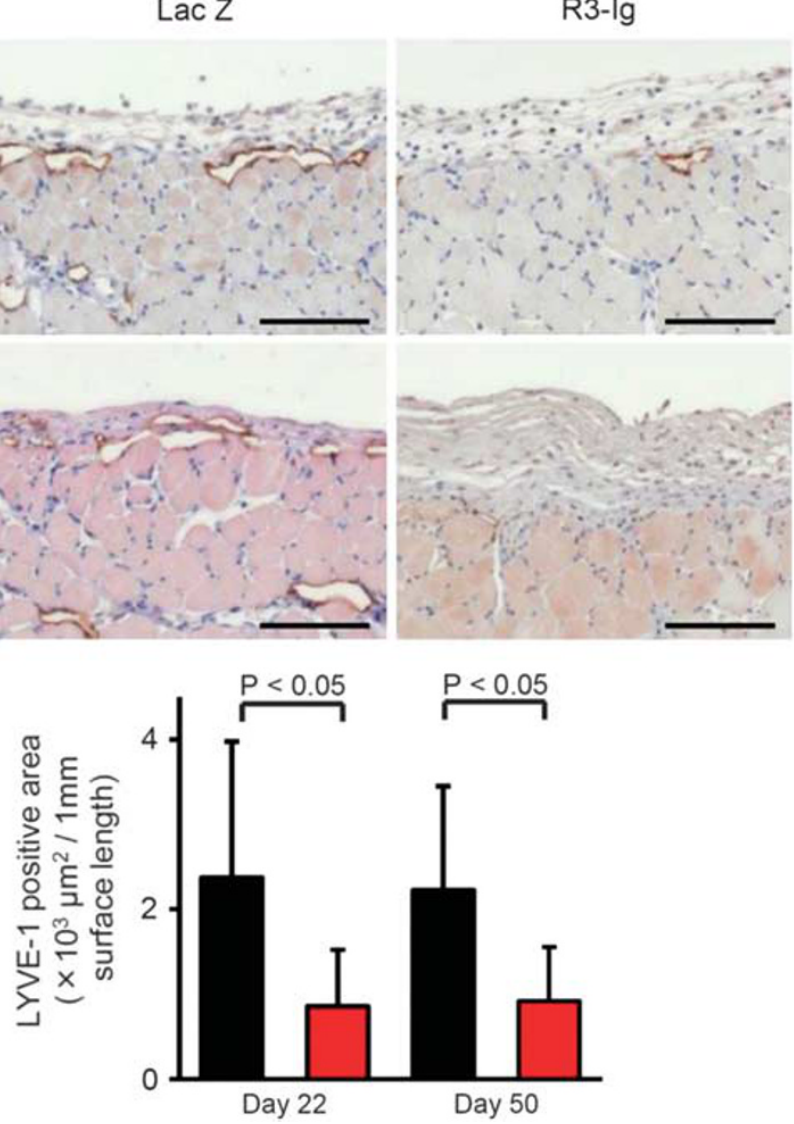

b

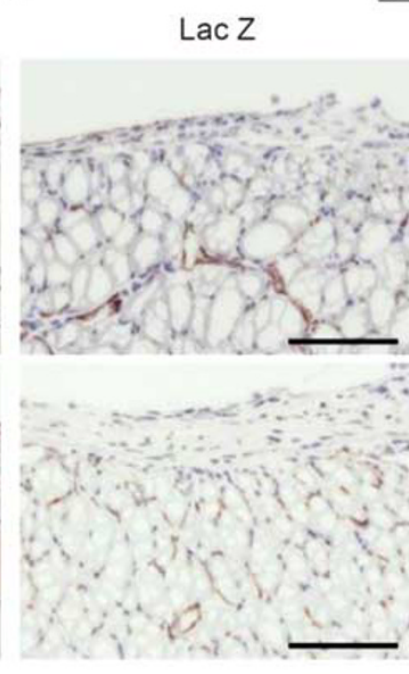

\section{$\underline{\text { CD31 }}$}

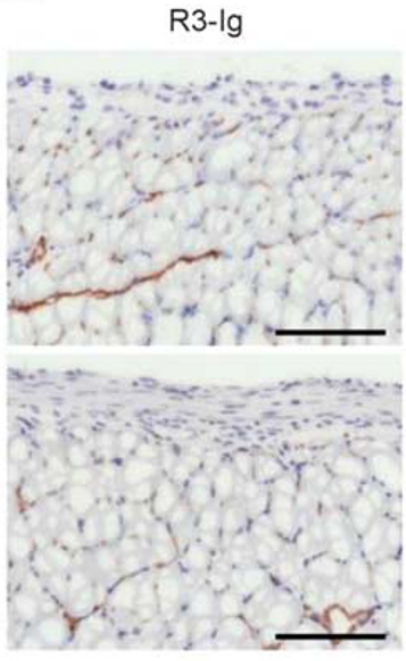

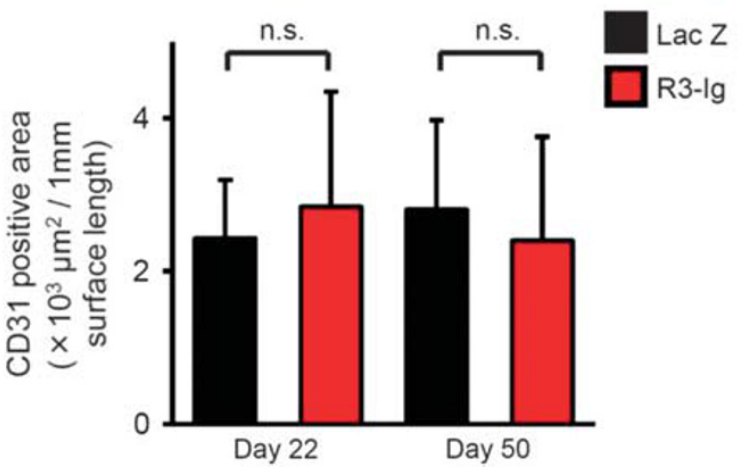

Figure 4 In immunohistochemical analyses of the diaphragm, adenovirus-expressing sVEGFR-3 suppressed LYVE-1-positive lymphatic vessels but not expression of CD31-positive vessels, type III collagen or CD68-positive cells. The diaphragm of mice treated with Adeno-Lac Z (Lac Z) or with AdenosVEGFR-3-lg (R3-lg) was immunohistochemically analyzed for: (a) LYVE-1-positive lymphatic vessels, (b) CD31-positive vessels, (c) deposition of type III collagen, and (d) CD68-positive macrophages. (each group, $n=6$ ). Scale bars, $100 \mu \mathrm{m}$. Lac Z: treatment with Adeno-LacZ, R3-Ig: treatment with AdenosVEGFR-3-Ig. LYVE-1, lymphatic vessel endothelial hyaluronan receptor-1; ND, not detected; NS, not significant; PD, peritoneal dialysis; RT-PCR, reverse transcription-PCR; sVEGFR-3, soluble vascular endothelial growth factor receptor-3. 

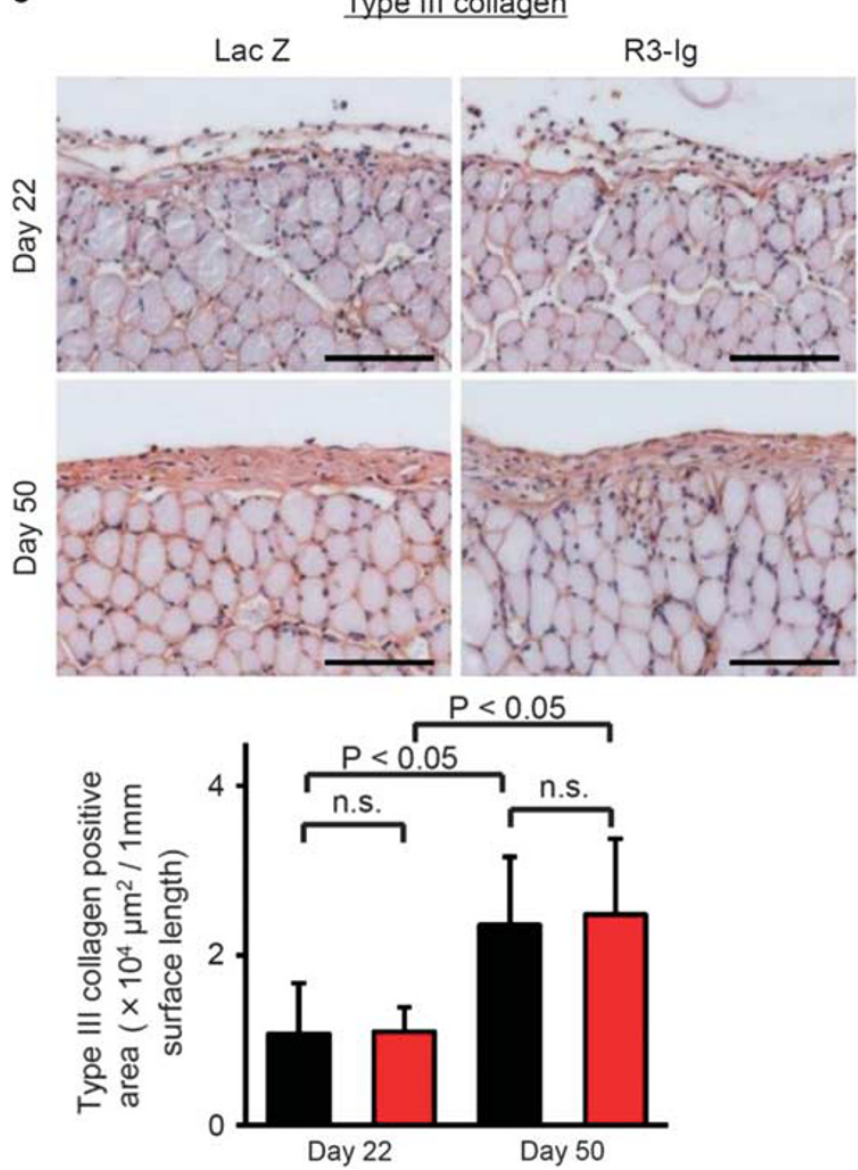

Figure 4 Continued.

analyses was similar between the Adeno-LacZ and AdenosVEGFR-3 groups (Figures $4 \mathrm{~b}$ and $5 \mathrm{e}$ ). Subsequently, we performed a conventional peritoneal equilibration test using a $4.25 \%$ glucose-based peritoneal dialysis fluid $(4.25 \% \mathrm{G}-$ peritoneal equilibration test, Dianeal) to assess peritoneal membrane transport. There was no difference in transport rates between the Adeno-LacZ and Adeno-sVEGFR-3 groups (Supplementary Figure 10a and b). On day 22, peritoneal inflammation associated with strong CD68-positive macrophage infiltration was shown (Figure 4d). It was thus considered that assessment of the peritoneal membrane function in mice was unsuitable under these conditions, as it is in human peritoneal dialysis patients with peritonitis. ${ }^{34}$ We therefore re-assessed the peritoneal equilibration test after resolution of inflammatory changes in the peritoneal membranes. For this purpose, after development of lymphangiogenesis in MGO models on day 22, the mice were observed for 4 weeks to watch for reduction in peritoneal inflammation (Experiment 3, Supplementary Figure 2c). However, we had difficulty in performing the peritoneal equilibration test on day 50 because there were several adhesions in the peritoneal cavity due to inflammation in the peritoneum (Supplementary Figure 11a). d

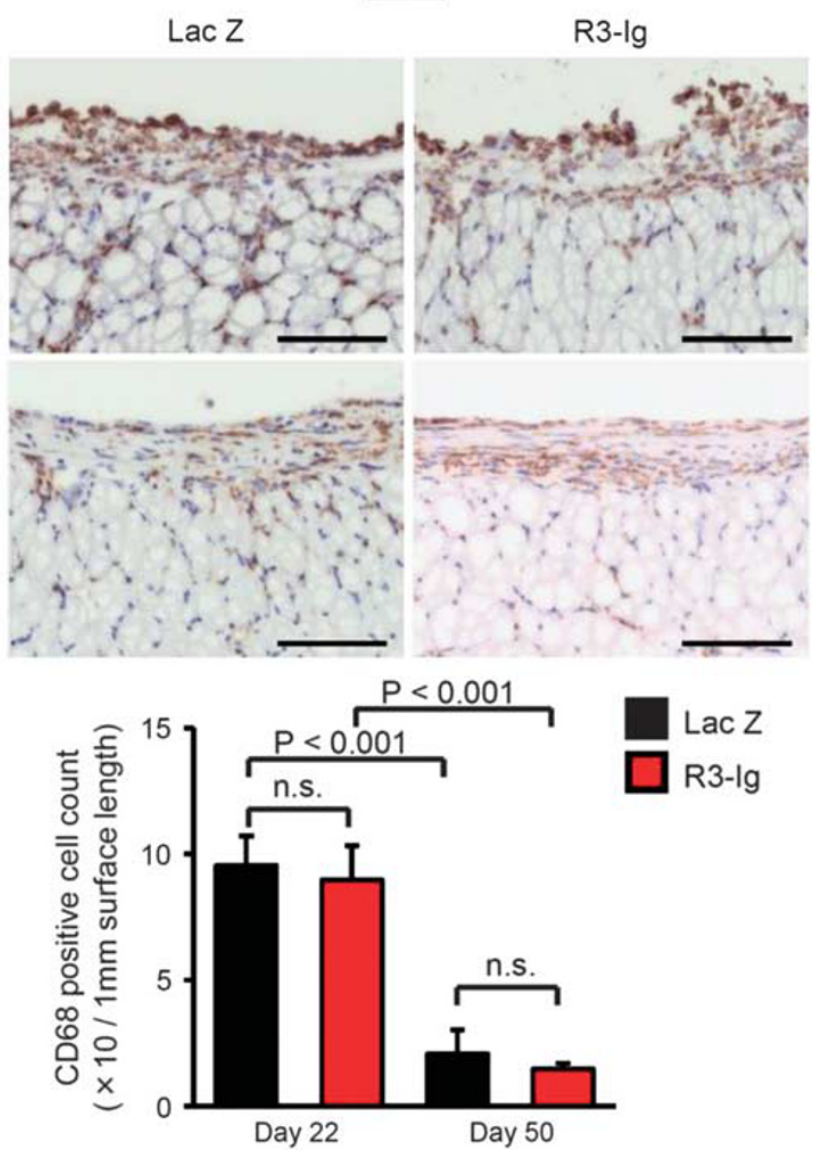

Therefore, to prevent adhesions and to reduce the peritoneal inflammation, we administered a low concentration (1.5\%) of a glucose-based peritoneal dialysis fluid into the peritoneal cavity 5 days per week from day 22 to day 50 (Experiment 4, Supplementary Figure 2d).

\section{Analysis of Pathology and Function in the Peritoneal Membrane on Day 50 After Administration of a $1.5 \%$ Glucose-Based Solution for $\mathbf{4}$ Weeks}

We found that peritoneal inflammation was successfully reduced by administration of the $1.5 \%$ glucose-based peritoneal dialysis fluid into the peritoneal cavity for 4 weeks, which enabled performance of a peritoneal equilibration test on day 50 (Supplementary Figure 11b). Leakage of PGE2 into the effluent, COX-2 mRNA expression, CD68-positive macrophages, and mast cell infiltration in the diaphragm were reduced on day 50 compared with day 22 in the AdenoLacZ and Adeno-sVEGFR-3 groups (Figures $4 \mathrm{~d}$ and 7). On day 50 , the lymphatic vessels that were suppressed on day 22 continued to be suppressed in the Adeno-sVEGFR-3 group, and both the lymphangiogenesis and neoangiogenesis conditions were preserved from day 22 to day 50 (Figure $4 \mathrm{a}$ and $\mathrm{b}$ ). 

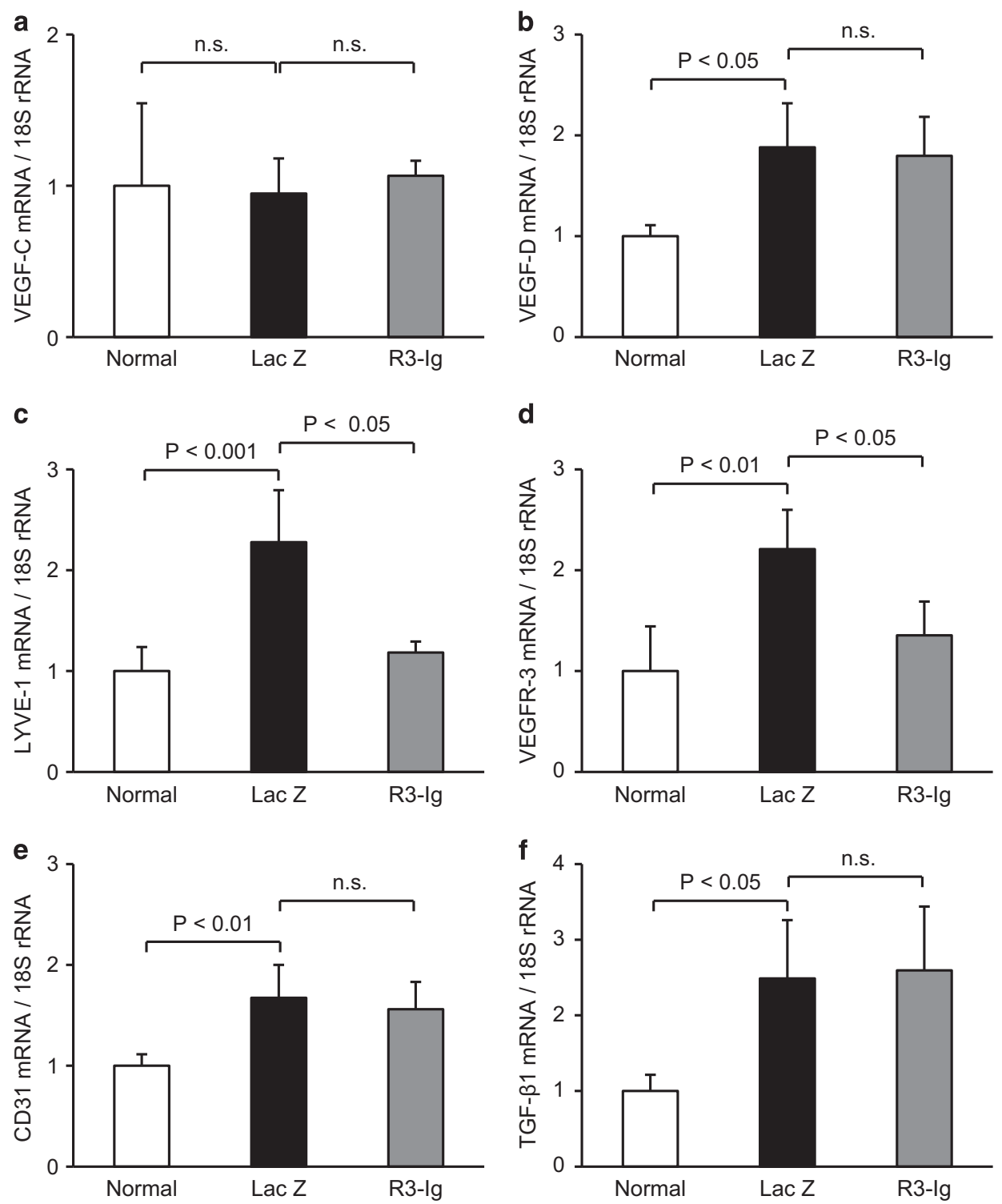

Figure 5 Quantitative PCR analysis of specific mRNA expression in the diaphragm of methylglyoxal mouse models indicated that adenovirus-sVEGFR-3 treatment suppressed lymphangiogenesis as assessed by LYVE-1 and VEGFR-3 mRNA expression; however, there was no difference between the AdenosVEGFR-3 and Adeno-LacZ groups in the expression of VEGF-C, -D, CD31, and TGF- $\beta 1$ mRNA (Day 22). (a) VEGF-C, (b) VEGF-D, (c) LYVE-1, (d) VEGFR-3, (e) CD31, and (f) TGF- $\beta 1$ mRNA expression in the diaphragms of the indicated mice. (each group, $n=6$ ). Lac $Z$, treatment with Adeno-LacZ; LYVE-1, lymphatic vessel endothelial hyaluronan receptor-1; NS, not significant; R3-Ig, treatment with Adeno-sVEGFR-3-Ig; sVEGFR-3, soluble vascular endothelial growth factor receptor-3; TGF- $\beta$, transforming growth factor-beta; VEGFR, vascular endothelial growth factor receptor.

Apparently, the progression of fibrosis as assessed by type III collagen expression was more advanced on day 50 compared with that on day 22; however, the extent of fibrosis was not different between the Adeno-sVEGFR-3 and Adeno-LacZ groups (Figure 4c). On day 50, we found a significant difference in fluid removal between the two groups in the $7.5 \%$ icodextrin peritoneal equilibration test (Figure 6a). This result indicates that the $7.5 \%$ icodextrin peritoneal equilibration test can assess fluid absorption via lymphatic vessels. In the $4.25 \% \mathrm{G}$-peritoneal equilibration test, we found a significant difference on day 22 between the control mice and the MGO model mice in the ratio of dialysate glucose at $2 \mathrm{~h}$ dwell time to dialysate glucose at $0 \mathrm{~h}$ dwell time; however, there was no difference on day 50, suggesting that the low levels on day 22 may be due to vascular permeability with strong inflammation (Figure 7 and Supplementary Figure 10a). In addition, there was no difference in the ratio of dialysate glucose at $2 \mathrm{~h}$ dwell time to dialysate glucose at $0 \mathrm{~h}$ or in the dialysate to plasma ratio of creatinine between the groups (Supplementary Figure 10a and b), indicating that lymphatic vessels are not involved in the function of solute transport. Similar tendencies in pathological findings were 
observed in the parietal peritoneal membrane by treatment with Adeno-sVEGFR-3 and Adeno-LacZ (Supplementary Figure 12). Amylase concentration in the effluent was not different between the two groups on day 22 or day 50 (Figure 6b).

\section{VEGF-D Concentration in Human Peritoneal Effluent Correlated with the Peritoneal Membrane Transport Rate. VEGF-D and VEGFR-3 mRNA Expression was Increased in Human Peritoneal Biopsy Samples of Ultrafiltration Failure}

In human peritoneal dialysis patients, VEGF-D concentration was correlated with the dialysate to plasma ratio of creatinine in the peritoneal dialysis effluent of samples dwelled for $4 \mathrm{~h}$ $(R=0.622, \quad P<0.001$, Figure $8 \mathrm{a})$. We further measured VEGF-D concentration in the overnight-dwelled peritoneal dialysis effluent of 83 patients; we found a positive correlation between the dialysate VEGF-D concentration and the dialysate to plasma ratio of creatinine $(R=0.516, P<0.001$, Figure $8 \mathrm{~b}$ ). We assessed the VEGF-D mRNA expression in the peritoneal membrane of human biopsy samples (Table 1). VEGF-D mRNA was significantly higher in the peritoneum of ultrafiltration failure patients than in the peritoneal membrane from patients on peritoneal dialysis without ultrafiltration failure or from patients with pre-dialysis uremia (Figure 8c). In ultrafiltration failure, VEGFR-3 and LYVE-1 mRNA expression was elevated compared with the other patients (Figure $8 \mathrm{~d}$ and Supplementary Figure 13). VEGF-D was mainly detected in CD68-positive macrophages of peritoneal biopsy samples of ultrafiltration failure patients (Figure 8e), which was similar to observations in the animal models (Figure $3 \mathrm{~b}$ and $\mathrm{c}$ ).

\section{DISCUSSION}

In the majority of organs, one of the main functions of lymphatic vessels is to return fluid and macromolecules to the vascular system. ${ }^{35,36}$ In peritoneal dialysis, the net ultrafiltration volume at the end of an exchange equals the cumulative net transcapillary water transport minus the lymphatic absorption during the exchange, ${ }^{37}$ therefore, control of lymphatic absorption is important to obtain higher drained volume. In a previous study using human materials and rat chlorhexidine gluconate models, we showed that lymphangiogenesis developed in the peritoneal cavity. ${ }^{5}$ In the present study, we demonstrated that lymphangiogenesis developed in rodent MGO models and found that lymphatic vessels grow in association with inflammatory changes, especially in the diaphragm. These findings and our previous studies $^{5}$ indicate that lymphangiogenesis is a common feature in the peritoneal cavity, together with inflammation and fibrosis, and that lymphangiogenesis is more predominant in the diaphragm compared with that in the parietal peritoneal membrane. Interestingly, in the MGO model animals on day 22, VEGF-D was significantly increased in the diaphragms of mice $(P<0.01$, Figure $2, P<0.05$, Supplementary Figure 5$)$ and rats $(P<0.05$, Supplementary Figure 6$)$; however, VEGF-C
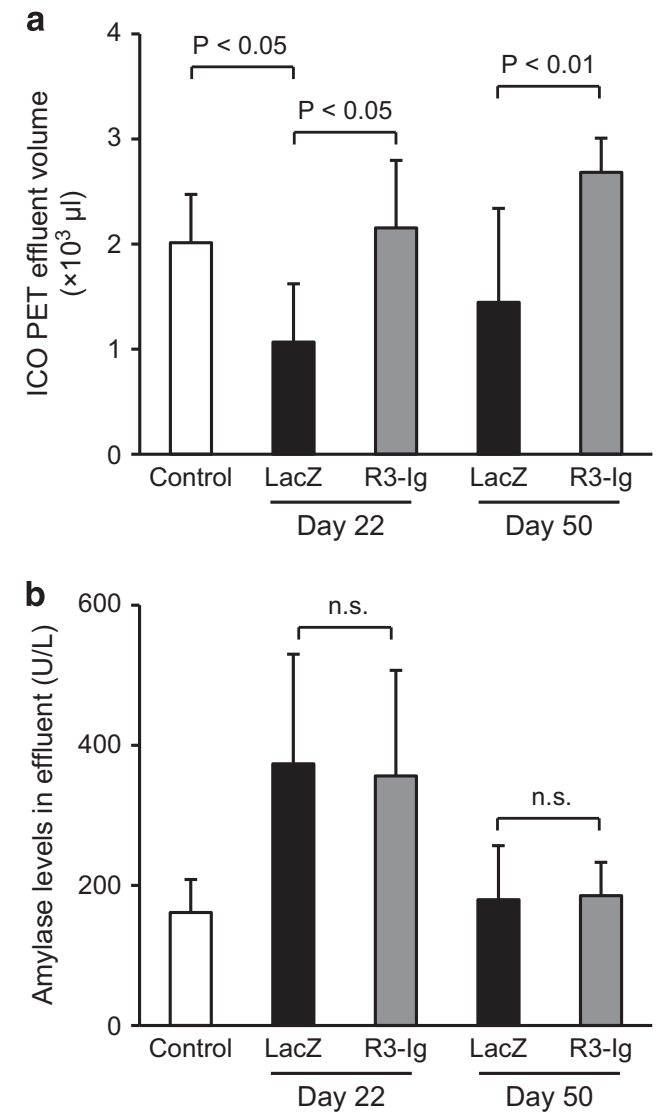

Figure 6 Analyses of peritoneal functions. Peritoneal equilibration test with a $7.5 \%$ icodextrin peritoneal dialysis fluid can identify the upregulation and suppression of lymphangiogenesis. Peritoneal dialysis fluid containing $7.5 \%$ icodextrin $(2000 \mu \mathrm{l})$ was intraperitoneally injected into control, Adeno-Lac Z, or Adeno-sVEGFR-3-Ig mice. Drained dialysate was collected $4 \mathrm{~h}$ after administration. (a) There was a difference in effluent volume between groups as detected by the $7.5 \%$ icodextrin peritoneal equilibration test, indicating that the $7.5 \%$ icodextrin peritoneal equilibration test can identify the suppression of lymphangiogenesis. (b) Amylase levels in the effluent were significantly increased on day 22 ; however, there was no difference between the groups on days 22 and 50. (each group, $n=6$ ). ICO PET, icodextrin peritoneal equilibration test; Lac Z, treatment with Adeno-LacZ; NS, not significant; R3-Ig, treatment with Adeno-sVEGFR-3-Ig; sVEGFR-3, soluble vascular endothelial growth factor receptor-3.

was not significantly upregulated (Figure 2, Supplementary Figures 5 and 6). This situation was different from that of the chlorhexidine gluconate models. ${ }^{5}$ In the chlorhexidine gluconate models of our previous studies, lymphangiogenesis was suppressed by COX-2 inhibitors; however, these drugs are not suitable for peritoneal dialysis patients in terms of preservation of residual renal function and are not suitable as specific inhibitors of lymphangiogenesis. ${ }^{5}$ VEGFR-3, a receptor for both VEGF-C and -D, is expressed by lymphatic endothelial cells and is upregulated in both MGO (Figure 2, Supplementary Figure 6) and chlorhexidine gluconate models $;{ }^{5}$ we therefore considered that VEGFR-3 is suitable as a target molecule to suppress lymphangiogenesis in the 
peritoneal cavity. The effect of sVEGFR-3 on inhibition of VEGFR-3 activation was similar to that of the VEGFR-3 kinase inhibitor in cultured human lymphatic endothelial cells stimulated with recombinant VEGF-D (Supplementary Figures 1 and 14).

In our previous studies, ${ }^{5}$ we used immunofluorescence microscopy to detect the passage of fluorescein isothiocyanate (FITC)-dextran (molecular weight 2000000 ), which can only be absorbed through the lymphatic vessels in the diaphragm, and detected high FITC-dextran levels in the serum of the chlorhexidine gluconate models. ${ }^{5}$ However, these methods are not appropriate for peritoneal dialysis patients. Therefore, we tried to develop practical methods using a 7.5\% icodextrin peritoneal dialysis solution, which reflects lymphatic functions. Icodextrin is a glucose polymer osmotic agent that is used to promote sustained ultrafiltration during long peritoneal dialysis dwells. ${ }^{33,38}$ The disappearance of icodextrin from the peritoneal cavity was reported to be consistent with a constant rate of fluid transport from the peritoneal cavity, mainly via the lymphatics. ${ }^{33}$ In our experiments, the drained volume of the $7.5 \%$ icodextrin peritoneal equilibration test identified the upregulation and suppression of lymphangiogenesis, even in the presence of inflammation on day 22 (Figure 6a). It has been reported that protein and solutions pass into the lymphatic capillaries without sieving ${ }^{14}$ because they have anatomically discontinuous basal lamina and have gaps between the lymphatic endothelial cells. ${ }^{6,35}$ This finding is in agreement with there being no difference in glucose and creatinine transport rates between the conditions of suppression and lack of suppression of lymphangiogenesis (Supplementary Figure 10). In the $4.25 \%$ G-peritoneal equilibration test, the drained volume tended to be increased in the Adeno-sVEGFR-3 groups, but the difference versus the volume in the Adeno-LacZ group was not statistically significant (Supplementary Figure 10c). This result suggests that the $4.25 \%$ G-peritoneal equilibration test may be unsuitable for identifying the different states of lymphatic absorption. In the use of icodextrin solution in rodents, the reduction in recovery volume was shown to result from degradation of icodextrin by 10- to 25 -fold higher levels of amylase compared with humans..$^{39,40}$ The low drained volume in the $7.5 \%$ icodextrin peritoneal equilibration test on day 22 was probably therefore due to high amylase concentrations (Figure 6). We did not find any differences in amylase concentrations in peritoneal dialysis effluent between the Adeno-sVEGFR-3 and Adeno-LacZ groups (Figure 6b). We performed the $7.5 \%$ icodextrin peritoneal equilibration test for a short duration of $4 \mathrm{~h}$, under which condition the removal of fluid from the circulation by the oncotic pressure of icodextrin solution may not be strongly affected. However,
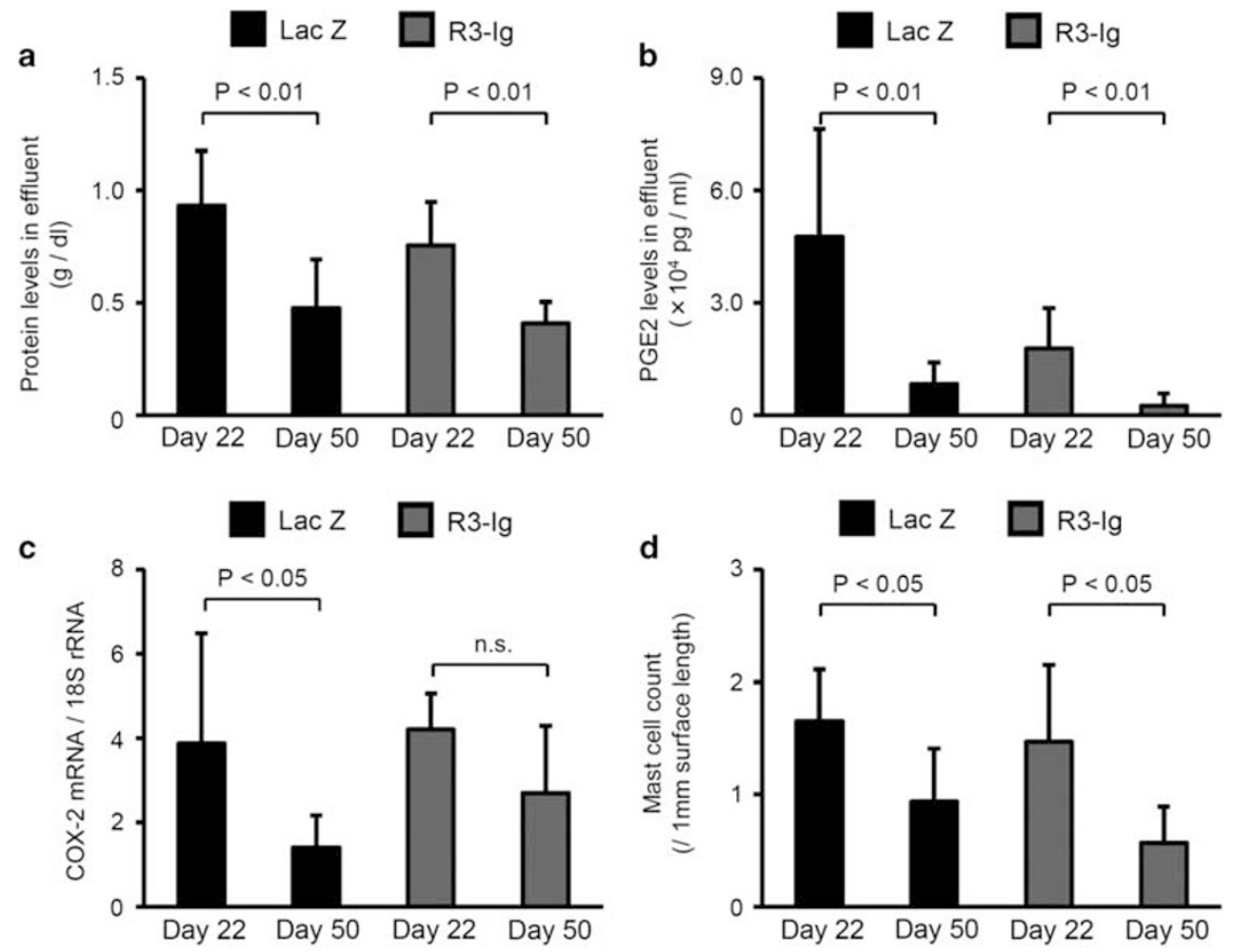

Figure 7 Peritoneal inflammation associated with protein and PGE2 leakage into peritoneal fluid. Increased expression of COX-2 mRNA and mast cells in diaphragms on day 22 was decreased on day 50 after peritoneal lavage. (a) Protein leakage into and (b) prostaglandin E2 (PGE2) in the peritoneal dialysis fluid. (c) COX-2 mRNA expression, and (d) the number of mast cells in the diaphragm. (each group, $n=6$ ). Lac $Z$, treatment with Adeno-LacZ; NS, not significant; R3-Ig, treatment with Adeno-sVEGFR-3-Ig. 
a

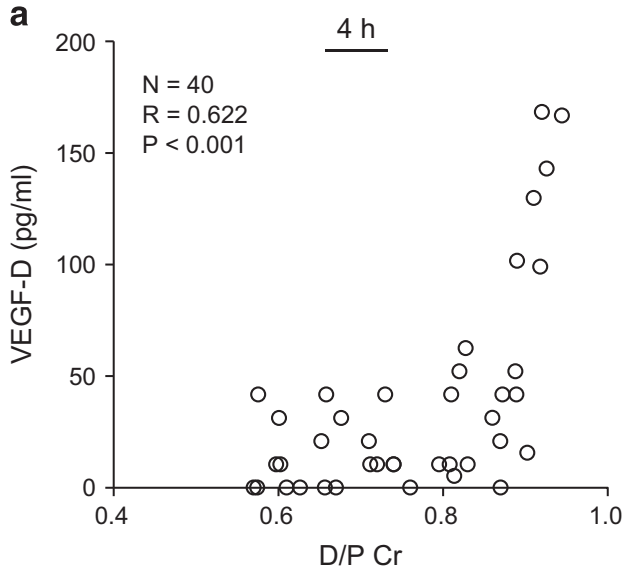

C

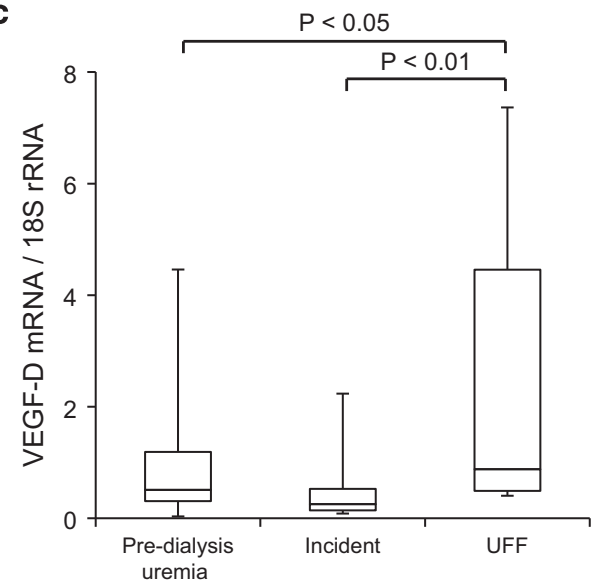

b

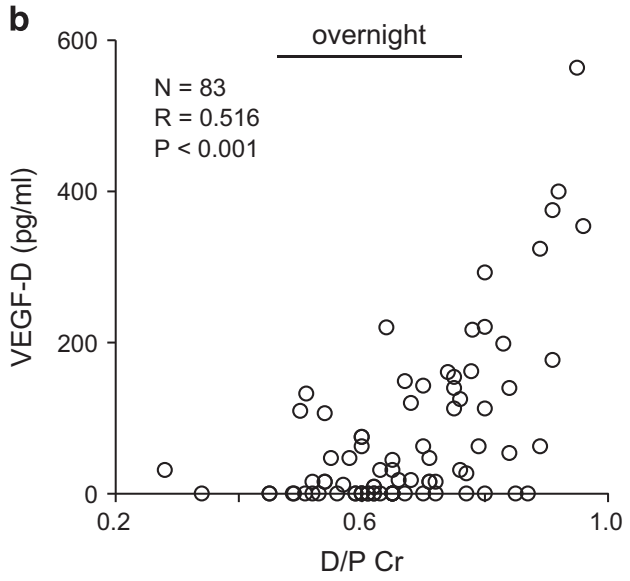

d

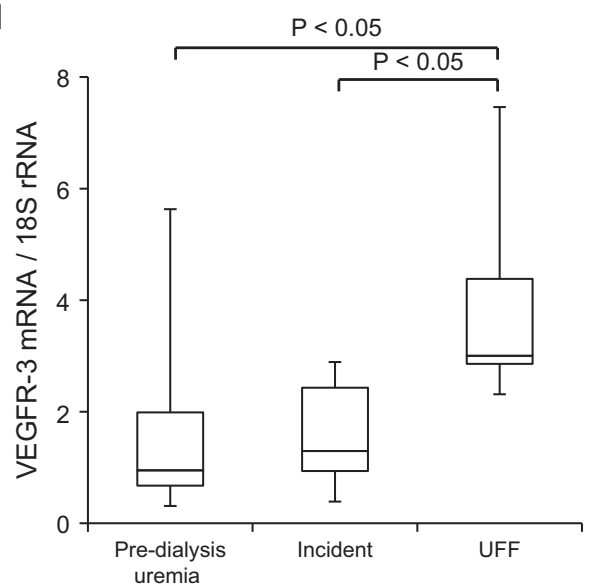

e

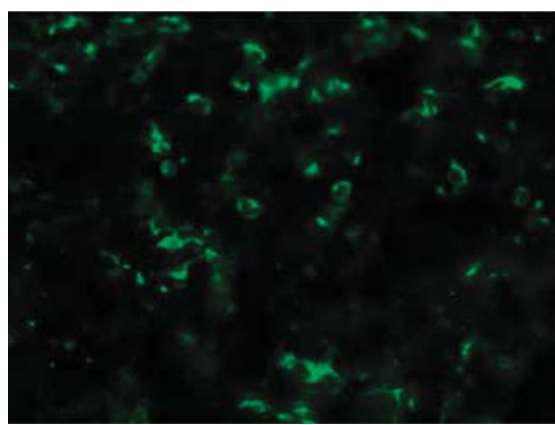

CD68

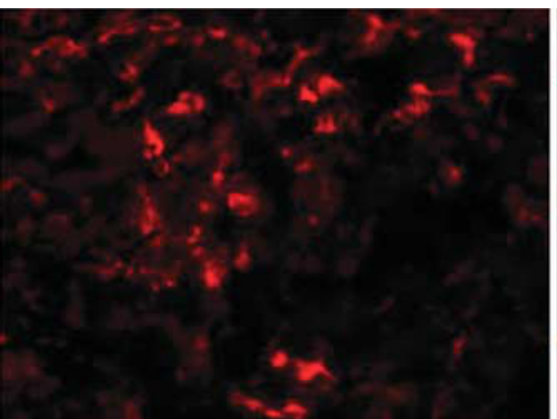

merge

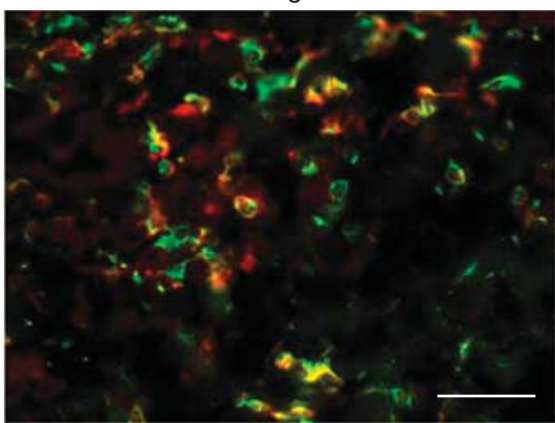

Figure 8 VEGF-D concentration in human peritoneal dialysis effluent correlated with the peritoneal transport rate (dialysate to plasma ratio of creatinine, D/P Cr). VEGF-D and VEGFR-3 mRNA levels were increased in ultrafiltration failure in human peritoneal biopsy samples. (a) Positive correlation between the VEGF-D concentration in the peritoneal dialysis effluent of samples dwelled for $4 \mathrm{~h}$ and the dialysate to plasma ratio of creatinine (D/P Cr). (b) Positive correlation between VEGF-D concentration in overnight-dwelled peritoneal dialysis effluent samples and the dialysate to plasma ratio of creatinine. VEGF-D (c) and VEGFR-3 (d) mRNA levels, as assessed using qRT-PCR, were significantly higher in patients with ultrafiltration failure than in patients with pre-dialysis uremia, or in patients in the incident group. (e) Double immunofluorescent analysis showed that VEGF-D is mainly expressed by macrophages in human peritoneal biopsy specimens of bacterial peritonitis. Scale bar, $50 \mu \mathrm{m}$. qRT-PCR, quantitative reverse transcription-PCR; UFF, ultrafiltration failure; VEGF, vascular endothelial growth factor.

to determine whether these methods might be available for peritoneal dialysis patients, future detailed studies, including studies of optimal conditions, are necessary.

We successfully evaluated peritoneal membrane function after peritoneal rest with lavage for reduction of inflammation. These results suggest that peritoneal lavage before removal of the peritoneal dialysis catheter after prolonged peritoneal dialysis treatment ${ }^{41}$ may be useful to prevent adhesions that could lead to encapsulating peritoneal sclerosis. 
In MGO model animals, VEGF-D that is expressed mainly by macrophages is a key growth factor for lymphangiogenesis in the peritoneal cavity, especially in the diaphragm (Figures $2 \mathrm{~b}$ and $3 \mathrm{~b}, \mathrm{c}$ and Supplementary Figure 6). In cultured macrophages and fibroblasts, VEGF-D has been reported to be increased by PGE2, ${ }^{42}$ which was elevated in the effluent of MGO model mice (Figure 7), and by inflammatory cytokines. ${ }^{43}$ In contrast to VEGF-C, VEGF-D was downregulated by TGF- $\beta{ }^{44}$ We have reported that mesothelial cells strongly express VEGF-C; ${ }^{5}$ however, VEGF-D was not expressed by mesothelial cells in the MGO model mice or in cultured human mesothelial cells (Figure $3 \mathrm{~d}$ and e). These instances of expression and regulation of VEGF-D are different from those of VEGF-C. We are the first to report that VEGF-D is increased in the human peritoneal membrane and in the peritoneal dialysis effluent with ultrafiltration failure (Figure 8). These data indicate that VEGF-D is involved in lymphangiogenesis in human peritoneal dialysis patients.

In summary, lymphatic absorption is a common feature associated with peritoneal inflammation and fibrosis, and VEGF-D is involved in the development of lymphangiogenesis. VEGFR-3 is a new target to increase net ultrafiltration by suppressing lymphatic absorption.

Supplementary Information accompanies the paper on the Laboratory Investigation website (http://www.laboratoryinvestigation.org)

\section{ACKNOWLEDGMENTS}

We express our gratitude to Mr Norihiko Suzuki, Ms Naoko Asano, Ms Keiko Higashide, and Ms Yuriko Sawa (Department of Nephrology and Renal Replacement Therapy, Nagoya University, Nagoya, Japan) for their technical assistance. We also thank Dr Makoto Mizutani (Handa Municipal Hospital, Handa, Japan), Dr Isao Ito (Yokkaichi Municipal Hospital, Yokkaichi, Japan), Dr Hirotake Kasuga (Nagoya Kyoritsu Hospital, Nagoya, Japan), Dr Takeyuki Hiramatsu (Kounan-Kousei Hospital, Kounan, Japan), and Dr Masanobu Horie (Daiyukai-Daiichi Hospital, Ichinomiya, Japan) for collecting peritoneum samples from the peritoneal dialysis patients. We also express our gratitude to Professor Kari Alitalo (University of Helsinki, Helsinki, Finland) and Dr Hajime Kubo (Kyoto University, Kyoto, Japan, currently working at Tanabe Mitsubishi Pharma Corporation, Osaka, Japan), and Vegenics Pty Limited (a subisidialy of Circadian Technologies Limited, Australia) for providing Adeno-VEGFR-3, and also Professor Alitalo and Dr Kubo for discussing the Adeno-sVEGFR-3 treatment and this manuscript. This work was supported in part by a Grant-inAid for Scientific Research from the Ministry of Education, Science, and Culture, Japan (YI \# 20590972), the 2013 research grant from the Aichi Kidney Foundation (T Terabayashi and YI), and the Japanese Association of Dialysis Physicians, Grant 2013-15 (YI). This study was also supported in part by a Grant-in-Aid for Progressive Renal Diseases Research, Research on Rare and Intractable Disease, from the Ministry of Health, Labor and Welfare of Japan.

\section{DISCLOSURE/CONFLICT OF INTEREST}

The authors declare no conflict of interest.

1. Mizuno M, Ito $Y$, Tanaka A et al. Peritonitis is still an important factor for withdrawal from peritoneal dialysis therapy in the Tokai area of Japan. Clin Exp Nephrol 2011;15:727-737.

2. Kawaguchi $Y$, Ishizaki T, Imada A et al. Searching for the reasons for drop-out from peritoneal dialysis: a nationwide survey in Japan. Perit Dial Int 2003;23 Suppl 2:S175-S177.
3. Williams JD, Craig KJ, Topley $\mathrm{N}$ et al. Morphologic changes in the peritoneal membrane of patients with renal disease. J Am Soc Nephrol 2002;13:470-479.

4. Mateijsen MA, van der Wal AC, Hendriks PM et al. Vascular and interstitial changes in the peritoneum of CAPD patients with peritoneal sclerosis. Perit Dial Int 1999;19:517-525.

5. Kinashi $\mathrm{H}$, Ito $\mathrm{Y}$, Mizuno $\mathrm{M}$ et al. TGF-beta1 promotes lymphangiogenesis during peritoneal fibrosis. J Am Soc Nephrol 2013;24: 1627-1642.

6. Sakamoto I, Ito Y, Mizuno M et al. Lymphatic vessels develop during tubulointerstitial fibrosis. Kidney Int 2009;75:828-838.

7. Suzuki $Y$, Ito $Y$, Mizuno $M$ et al. Transforming growth factor-beta induces vascular endothelial growth factor- $C$ expression leading to lymphangiogenesis in rat unilateral ureteral obstruction. Kidney Int 2012;81:865-879.

8. Lee $A S$, Lee JE, Jung $\mathrm{YJ}$ et al. Vascular endothelial growth factor-C and $-D$ are involved in lymphangiogenesis in mouse unilateral ureteral obstruction. Kidney Int 2013;83:50-62.

9. Smit W, Schouten N, van den Berg N et al. Analysis of the prevalence and causes of ultrafiltration failure during long-term peritoneal dialysis: a cross-sectional study. Perit Dial Int 2004;24:562-570.

10. Sampimon DE, Coester AM, Struijk DG et al. The time course of peritoneal transport parameters in peritoneal dialysis patients who develop encapsulating peritoneal sclerosis. Nephrol Dial Transplant 2011;26:291-298.

11. Heimburger O, Waniewski J, Werynski A et al. Peritoneal transport in CAPD patients with permanent loss of ultrafiltration capacity. Kidney Int 1990;38:495-506.

12. Fussholler A, zur Nieden S, Grabensee B et al. Peritoneal fluid and solute transport: influence of treatment time, peritoneal dialysis modality, and peritonitis incidence. J Am Soc Nephrol 2002;13: 1055-1060.

13. Krediet RT. The effective lymphatic absorption rate is an accurate and useful concept in the physiology of peritoneal dialysis. Perit Dial Int 2004;24:309-313 discussion 316-307.

14. Flessner M. Effective lymphatic absorption rate is not a useful or accurate term to use in the physiology of peritoneal dialysis. Perit Dial Int 2004;24:313-316.

15. Hirahara I, Kusano E, Yanagiba $\mathrm{S}$ et al. Peritoneal injury by methylglyoxal in peritoneal dialysis. Perit Dial Int 2006;26:380-392.

16. Hirahara I, Ishibashi $Y$, Kaname $S$ et al. Methylglyoxal induces peritoneal thickening by mesenchymal-like mesothelial cells in rats. Nephrol Dial Transplant 2009;24:437-447.

17. Kitamura $M$, Nishino $T$, Obata $Y$ et al. Epigallocatechin gallate suppresses peritoneal fibrosis in mice. Chem Biol Interact 2012;195: 95-104.

18. Nakayama K, Nakayama M, Iwabuchi $M$ et al. Plasma alpha-oxoaldehyde levels in diabetic and nondiabetic chronic kidney disease patients. Am J Nephrol 2008;28:871-878.

19. He Y, Kozaki K, Karpanen T et al. Suppression of tumor lymphangiogenesis and lymph node metastasis by blocking vascular endothelial growth factor receptor 3 signaling. J Natl Cancer Inst 2002;94:819-825.

20. He Y, Rajantie I, Pajusola K et al. Vascular endothelial cell growth factor receptor 3-mediated activation of lymphatic endothelium is crucial for tumor cell entry and spread via lymphatic vessels. Cancer Res 2005;65: 4739-4746.

21. Yang H, Kim C, Kim MJ et al. Soluble vascular endothelial growth factor receptor-3 suppresses lymphangiogenesis and lymphatic metastasis in bladder cancer. Mol Cancer 2011;10:36.

22. Kim YL, Kim SH, Kim JH et al. Effects of peritoneal rest on peritoneal transport and peritoneal membrane thickening in continuous ambulatory peritoneal dialysis rats. Perit Dial Int 1999;19(Suppl 2): S384-S387.

23. Mizutani $M$, Ito $\mathrm{Y}$, Mizuno $\mathrm{M}$ et al. Connective tissue growth factor (CTGF/CCN2) is increased in peritoneal dialysis patients with high peritoneal solute transport rate. Am J Physiol Renal Physiol 2010;298: F721-F733.

24. Nishimura $\mathrm{H}$, Ito $\mathrm{Y}$, Mizuno $\mathrm{M}$ et al. Mineralocorticoid receptor blockade ameliorates peritoneal fibrosis in new rat peritonitis model. Am J Physiol Renal Physiol 2008;294:F1084-F1093.

25. Makinen $T$, Jussila $L$, Veikkola $T$ et al. Inhibition of lymphangiogenesis with resulting lymphedema in transgenic mice expressing soluble VEGF receptor-3. Nat Med 2001;7:199-205. 
26. Twardowski ZJ. The fast peritoneal equilibration test. Semin Dial 1990;3:141-142.

27. Honda K, Hamada C, Nakayama M et al. Impact of uremia, diabetes, and peritoneal dialysis itself on the pathogenesis of peritoneal sclerosis: a quantitative study of peritoneal membrane morphology. Clin J Am Soc Nephrol 2008;3:720-728.

28. Takei $Y$, Kadomatsu K, Yuzawa $Y$ et al. A small interfering RNA targeting vascular endothelial growth factor as cancer therapeutics. Cancer Res 2004;64:3365-3370.

29. Yung S, Li FK, TM C. Peritoneal mesothelial cell culture and biology. Perit Dial Int 2006;26:162-173.

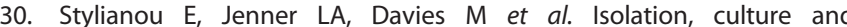
characterization of human peritoneal mesothelial cells. Kidney Int 1990;37:1563-1570.

31. Onogawa S, Kitadai Y, Tanaka $S$ et al. Expression of VEGF-C and VEGF-D at the invasive edge correlates with lymph node metastasis and prognosis of patients with colorectal carcinoma. Cancer Sci 2004;95: 32-39.

32. Nykanen Al, Sandelin H, Krebs $\mathrm{R}$ et al. Targeting lymphatic vesse activation and CCL21 production by vascular endothelial growth factor receptor-3 inhibition has novel immunomodulatory and antiarteriosclerotic effects in cardiac allografts. Circulation 2010;121:1413-1422.

33. Moberly JB, Mujais S, Gehr T et al. Pharmacokinetics of icodextrin in peritoneal dialysis patients. Kidney Int 2002;62:S23-S33.

34. Peritoneal Dialysis Adequacy 2006 Work Group. Clinical practice guidelines for peritoneal adequacy, update 2006. Am J Kidney Dis 2006:48:S91-S175.

35. Tammela T, Alitalo K. Lymphangiogenesis: Molecular mechanisms and future promise. Cell 2010;140:460-476.
36. Alitalo K, Tammela T, Petrova TV. Lymphangiogenesis in development and human disease. Nature 2005;438:946-953.

37. Mactier RA, Khanna R, Twardowski Z et al. Contribution of lymphatic absorption to loss of ultrafiltration and solute clearances in continuous ambulatory peritoneal dialysis. J Clin Invest 1987:80:1311-1316.

38. Johnson DW, Agar J, Collins J et al. Recommendations for the use of icodextrin in peritoneal dialysis patients. Nephrology (Carlton) 2003;8: $1-7$.

39. Garcia-Lopez E, Pawlaczyk K, Anderstam B et al. Icodextrin metabolism and alpha-amylase activity in nonuremic rats undergoing chronic peritoneal dialysis. Perit Dial Int 2007;27:415-423.

40. Pawlaczyk K, Garcia-Lopez E, Kuzlan-Pawlaczyk M et al. The effect of icodextrin-based solutions on peritoneal transport in rats undergoing chronic peritoneal dialysis. Perit Dial Int 2001;21(Suppl 3):S359-S361.

41. Yamamoto $T$, Nagasue $K$, Okuno $S$ et al. The role of peritoneal lavage and the prognostic significance of mesothelial cell area in preventing encapsulating peritoneal sclerosis. Perit Dial Int 2010;30:343-352.

42. Hosono $\mathrm{K}$, Suzuki T, Tamaki $\mathrm{H}$ et al. Roles of prostaglandin E2-EP3/EP4 receptor signaling in the enhancement of lymphangiogenesis during fibroblast growth factor-2-induced granulation formation. Arterioscler Thromb Vasc Biol 2011;31:1049-1058.

43. Watari K, Nakao S, Fotovati A et al. Role of macrophages in inflammatory lymphangiogenesis: Enhanced production of vascular endothelial growth factor C and D through NF-kappaB activation. Biochem Biophys Res Commun 2008;377:826-831.

44. Cui Y, Osorio JC, Risquez $C$ et al. Transforming growth factor- $\beta 1$ downregulates vascular endothelial growth factor-D expression in human lung fibroblasts via JNK signaling pathway. Mol Med 2014;20: $120-134$. 